\title{
The Grainyhead/LSF transcription factor GRH-1 is rhythmically required for molting
}

\author{
Milou W.M. Meeuse ${ }^{1,2}$, Smita Nahar ${ }^{1}$, Kathrin Braun ${ }^{1}$, Helge Großhans ${ }^{1,2,3}$ \\ ${ }^{1}$ Friedrich Miescher Institute for Biomedical Research (FMI), Maulbeerstrasse 66, CH-4058 Basel \\ ${ }^{2}$ University of Basel, Petersplatz 1, CH-4001 Basel \\ ${ }^{3}$ Correspondence to: helge.grosshans@fmi.ch
}

\begin{abstract}
Molting, that is, the synthesis and shedding of a cuticular exoskeleton, is a defining characteristic of ecdysozoa. In nematodes such as C. elegans, molts rhythmically terminate each of four larval stages. The molting cycle is tightly coupled to the rhythmic expression of thousands of genes. The mechanisms that support the regular molting cycle and oscillatory gene expression have remained largely elusive. Here, we performed an RNAi-based screen for transcription factors required for molting to identify potential components of a molting clock. We find that depletion of GRH-1, BLMP-1, NHR-23, NHR-25, MYRF-1 or BED-3 impairs progression through the molting cycle. We characterize GRH-1, a Grainyhead/LSF transcription factor whose functions in C. elegans development have remained largely unexplored, but whose orthologues in other animals are known to be key epithelial cell fate regulators. We show that GRH-1 depletion causes a dose-dependent extension of molt duration, with severe depletion causing defects in cuticle formation and shedding, and larval death. GRH-1 is required repetitively, during a specific time window prior to each larval molt. This rhythmic activity is consistent with the rhythmic accumulation of GRH-1 protein. These features are consistent with GRH-1 functioning as a key component, or high-level output, of the a gene regulatory network that controls molting. As its mammalian orthologues, as well as those of BLMP-1 and NHR-23, have been implicated in rhythmic homeostatic skin regeneration in mouse, the mechanisms underlying rhythmic $C$. elegans molting may be conserved beyond nematodes.
\end{abstract}




\section{Introduction}

C. elegans larval development subdivides into four larval stages, each terminated by a molt. In a first step, termed apolysis, the connections of the existing cuticle to the underlying epidermis are severed. Subsequently, a new cuticle is synthesized, before the old cuticle is shed in a final step termed ecdysis. Traditionally, the time of molting is equated with lethargus, a period of relative behavioral quiescence, when animals stop feeding. For simplicity we will follow this tradition here, but note that additional events required for successful molting precede lethargus (Cohen et al., 2020; Cohen and Sundaram, 2020); (Tsiairis and Großhans, 2021).

Molts occur with a regular period of $7-8 \mathrm{hr}$ at $25^{\circ} \mathrm{C}$, and a clock-type mechanism has been invoked to explain this regularity (Monsalve and Frand, 2012; Tsiairis and Großhans, 2021). Such a clock mechanism may also explain, and be partially based on, the rhythmic expression of thousands of genes that is coupled to the molting cycle (Hendriks et al., 2014; Kim et al., 2013; Meeuse et al., 2020; Turek and Bringmann, 2014). However, the components of this clock, and accordingly their wiring, have remained largely elusive (Tsiairis and Großhans, 2021).

Recently, we showed that rhythmic RNA polymerase II recruitment drives RNA level oscillations during larval development (Hauser et al., 2021). This finding suggests that rhythmically active transcription factors are components of the underlying machinery, or core oscillator, and thus presumably also the molting cycle clock. To identify possible candidates, we screened through a selection of rhythmically expressed transcription factors (TFs), assuming that rhythmic transcription would be a parsimonious (though not necessarily the only) mechanism of achieving rhythmic transcription factor activity. From a set of 92 such transcription factors (Hendriks et al., 2014), we sought to identify those that affected duration of, or progression through, molts. We identified six hits, including the nuclear hormone receptors NHR-23 and NHR-25 that were previously linked to molting (Frand et al., 2005; Gissendanner et al., 2004; Gissendanner and Sluder, 2000; Johnson et al., 2021; Kostrouchova et al., 1998; Kostrouchova et al., 2001). We characterize the function of $\mathrm{GRH}-1$, the sole $C$. elegans member of the phylogenetically conserved LSF/Grainyhead family (Venkatesan et al., 2003).

Grainyhead proteins are key regulators of epithelial cell fates that function in differentiation, maintenance, integrity and repair of different epithelial tissues in animals (Sundararajan et al., 2020). C. elegans GRH-1 was previously found to be required for embryonic cuticle formation, but its postembryonic function and regulation have remained unknown. We report that GRH-1 accumulates 
rhythmically and promotes molting through its activity in a specific window during each larval stage. Its depletion delays the onset of ecdysis in a dose-dependent manner to the point that animals severely depleted arrest development and die by bursting through a defective cuticle. The results, together with the validation of an additional hit, BLMP-1, in separate work by us and others (Hauser et al., 2021; Stec et al., 2021) provide new insights into the transcriptional mechanisms that support rhythmic molting and identifies potential molting clock components. The fact that proteins orthologous to GRH-1 as well as to additional screen hits also function in rhythmic homeostatic skin regeneration in mouse (Magnusdottir et al., 2007; Steinmayr et al., 1998; Telerman et al., 2017; Wilanowski et al., 2008) suggest mechanistic similarities between this process and the molting process of nematodes.

\section{Results}

\section{A targeted screen identifies transcription factors involved in molting}

Since C. elegans molting cycles are tightly coupled to oscillatory gene expression (Meeuse et al., 2020), which in turn is driven by rhythmic transcription (Hauser et al., 2021), we sought to identify rhythmically active transcription factors involved in molting. Hence, we performed an RNAi screen targeting 92 transcription factors that exhibit transcript level oscillations according to our previous annotation (Hendriks et al., 2014). Specifically, we screened for aberrant developmental progression or molt execution. To obtain such information, we examined luciferase activity in animals that express a luciferase transgene and that are grown in the presence of D-luciferin (Meeuse et al., 2020; Olmedo et al., 2015). This assay detects lethargus by a drop in luminescence at the level of individual animals, allowing us to quantify durations of molts, intermolts and, as a sum of the two, entire larvael stages for several animals per condition. We depleted the transcription factors by feeding animals on RNAi-expressing bacteria. To control for differences in larval growth among RNAi conditions unrelated to target protein depletion, we performed the experiment in parallel on RNAi-deficient $r d e-1$ (ne219) mutant animals (Tabara et al., 1999) (Figure 1A).

We expected mutants with the following phenotypes: i) altered developmental tempo, which could be faster or slower, and specific for a particular oscillation phase or continuously through the cycle, ii) increased variability of developmental tempo among individuals, and iii) cessation of development and/or failed molts. 
By plotting the luminescence intensities sorted by entry into the first molt in a heatmap (Figure 1B,C), we identified six genes whose depletion caused abnormal progression through development following atypical molts ( $n h r-23$, myrf-1 and grh-1; Figure 1B,D, Figure S1), or aberrant duration of molts (bed-3, blmp-1 and nhr-25; Figure 1C,D, Figure S2).

The nuclear hormone receptors NHR-23 and NHR-25 have previously been shown to function in molting (Frand et al., 2005; Gissendanner et al., 2004; Gissendanner and Sluder, 2000; Johnson et al., 2021; Kostrouchova et al., 1998; Kostrouchova et al., 2001), and we and others have described functions of BLMP-1 in oscillatory gene expression and cuticle formation (Hauser et al., 2021; Sandhu et al., 2021; Stec et al., 2021). Here, we sought to establish further the function of GRH-1, first, because we wished to understand whether class III phenotypes can indeed be predictive of clock dysfunction, second, because orthologous Grainyhead(-like) proteins function in epithelial cell fate control and remodeling of epithelial tissues in other animals (Sundararajan et al., 2020), suggesting a potentially conserved function and third, because its function in C. elegans development has remained largely unexplored (Venkatesan et al., 2003).
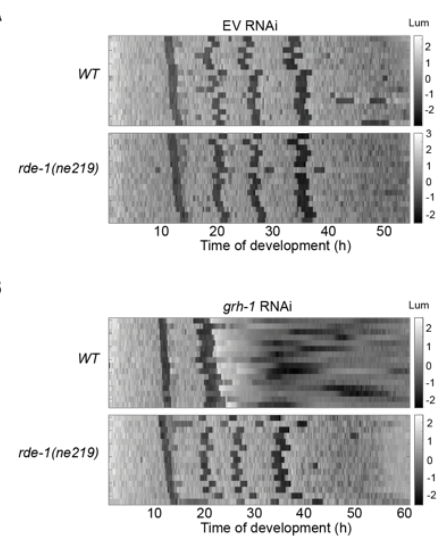

myrt-1 RNAi
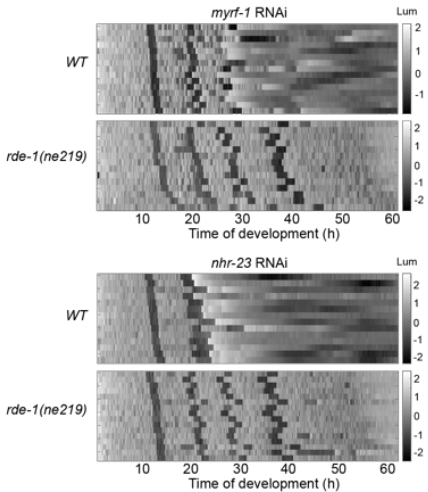
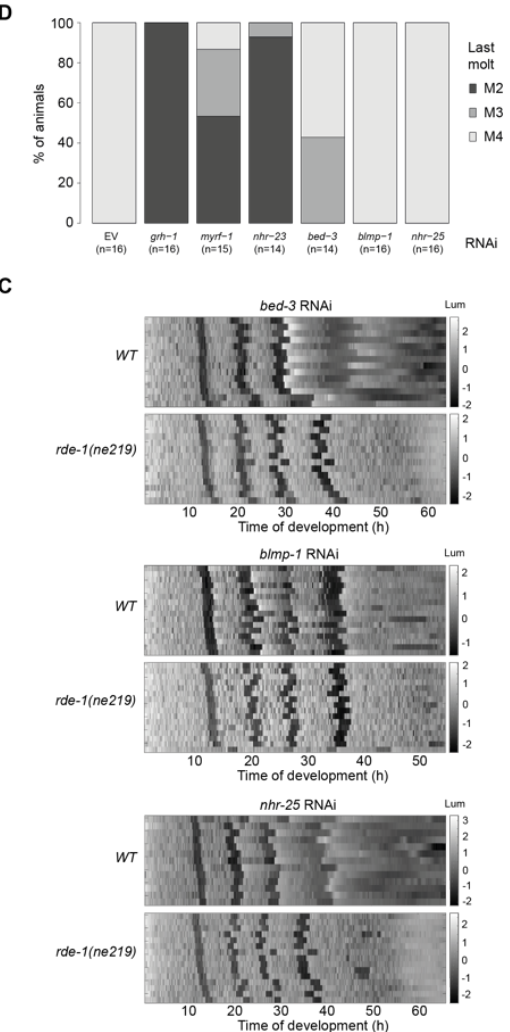

Figure 1: RNAi screen for 'oscillating' transcription factors revealed aberrant duration or progression of molting

A, Heatmap showing trend-corrected luminescence (Lum) of wild-type (WT, strain HW1939, top) and RNAi-deficient (rde-1(ne219), strain HW2150, bottom) animals expressing luciferase from the eft-3 promoter grown on mock 
(empty vector, EV) RNAi in a temperature-controlled incubator set to $20^{\circ} \mathrm{C}$. Each line represents one animal. Hatch is set to $t=0$ hours and traces are sorted by entry into the first molt. Darker color corresponds to low luminescence and is associated with the molt.

B, C Heatmap showing trend-corrected luminescence as in A, for indicated RNAi conditions causing altered numbers (B) or durations (C) of molts, respectively.

D, Quantification of the percentage of animals entering specific molts on indicated RNAi conditions. Shown are the last molts observed for animals in each condition; e.g., $100 \%$ of GRH-1 depleted fail to progress beyond M2.

\section{GRH-1 is repetitively required for normal duration and progression through molts}

Injection of double-stranded RNA targeting grh-1 into the germline of L4 stage larval animals causes embryonic lethality in the next generation (Venkatesan et al., 2003). Consistent with this finding, we observed a penetrant embryonic lethality phenotype in $g r h-1(0)$ mutant animals (data not shown). To bypass this defect and investigate the role of GRH-1 during larval development, we performed controlled GRH-1 depletion in larvae using the auxin-inducible degradation (AID) system (Zhang et al., 2015). We tagged grh-1 endogenously with degron::3xflag to generate allele grh-1(xe135), and expressed the plantspecific F-box protein TIR1 as a transgene, generating a strain that, for simplicity's sake, we will refer to as aid::grh-1 in the following. When we hatched aid::grh-1 embryos in the presence of $250 \mu \mathrm{M}$ auxin, animals failed to develop beyond the first molt (M1), i.e., none of them entered M2 (Figure 2A).

If GRH-1 were a component of the core molting machinery, we would predict that it be required for each molt. Indeed, when we depleted GRH-1 by addition of auxin at the beginning of the L2, L3 and L4 stage, respectively, animals in each case failed to develop beyond the next molt, i.e., M2, M3 and M4, respectively (Figure 2B-D). We conclude that GRH-1 is repetitively required during development, for completion of each molt. 
A

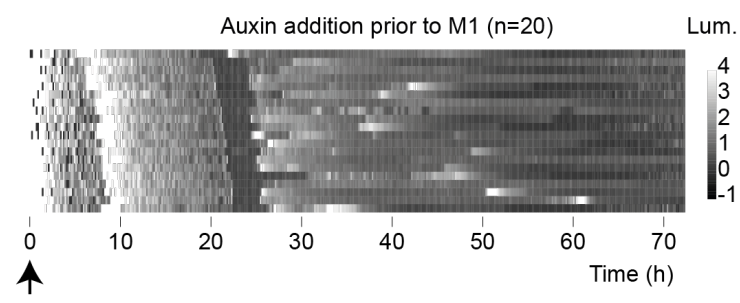

C

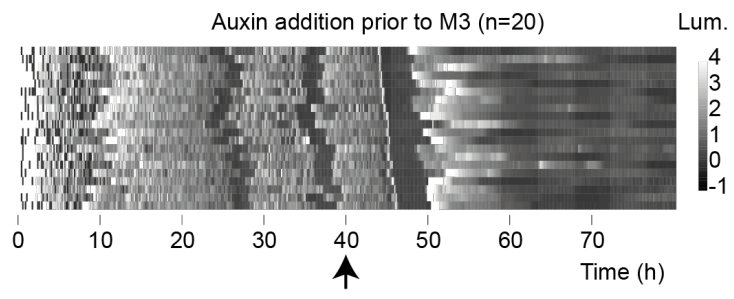

B

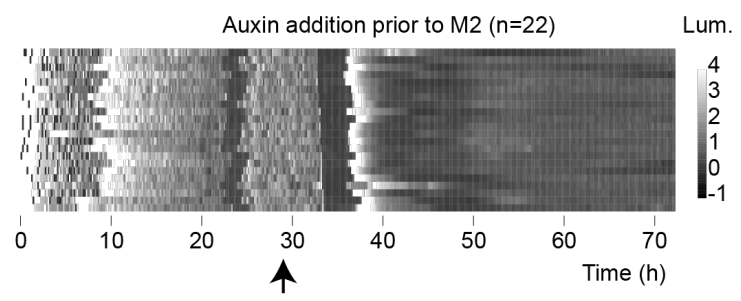

D

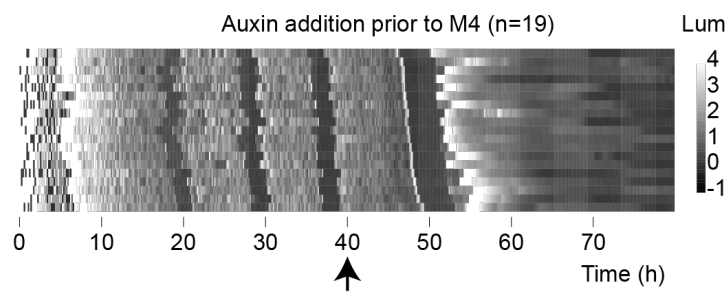

Figure 2: GRH-1 is required for ecdysis of every molt.

A-D, Heatmaps showing trend-corrected luminescence (Lum) of aid::grh-1 animals constitutively expressing luciferase (HW2434). $\mathrm{t}=0 \mathrm{~h}$ corresponds to time of plating embryos, which subsequently hatch at different times. Arrow indicates time point when $250 \mu \mathrm{M}$ auxin was added, i.e. prior to the first molt (A; M1), M2 (B), M3 (C) or M4 (D) larval stage. Note that for technical convenience in (A), auxin was provided at time of plating. Animals are sorted by entry into M1 (A), M2 (B), M3 (C), M4 (D), respectively. Note that embryos in (C) were younger than those in (D) when plated so that addition of auxin $40 \mathrm{~h}$ after plating coincides with $\mathrm{L} 3$ in (C) but L4 in (D).

We observed an increased duration of the last observed molt that GRH-1-depleted animals entered (Figure 2). To examine this phenotype further, we exposed aid::grh-1 animals to varying auxin concentrations to titrate $\mathrm{GRH}-1$ depletion. Although auxin concentrations of $\geq 400 \mathrm{nM}$ at hatching yielded a fully penetrant M1 exit phenotype, a dose-dependent decrease in phenotype penetrance occurred below this concentration (Figure 3A, S3A). When we quantified the developmental tempo for animals that, at the lowest auxin concentrations tested (53 nM, $79 \mathrm{nM}$ and $119 \mathrm{nM}$ ), completed the first three molts, we observed a dose-dependent lengthening in M1, M2 and M3 (Figure 3B, S3 - S5). By contrast, no or little change occurred for the duration of the intermolts preceding the lengthened molts (Figure 3C, S3 - S5). Hence, we conclude that the activity of GRH-1 is required for normal duration of the recurring molts, with strong depletion additionally causing molting failure. 


\section{A}
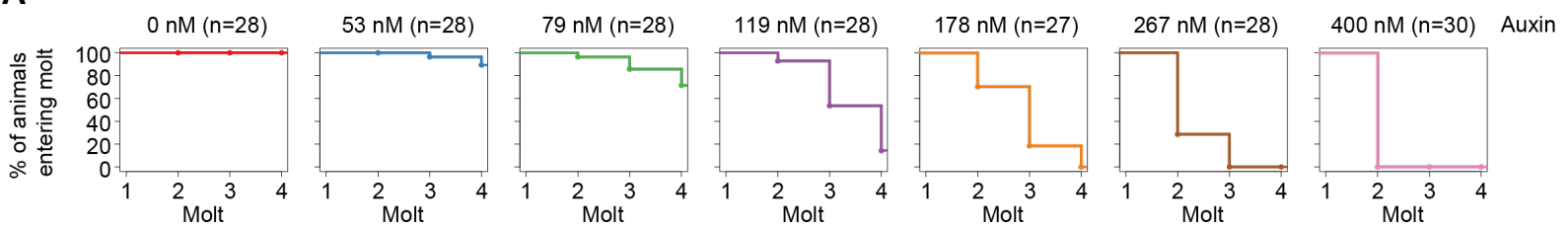

B
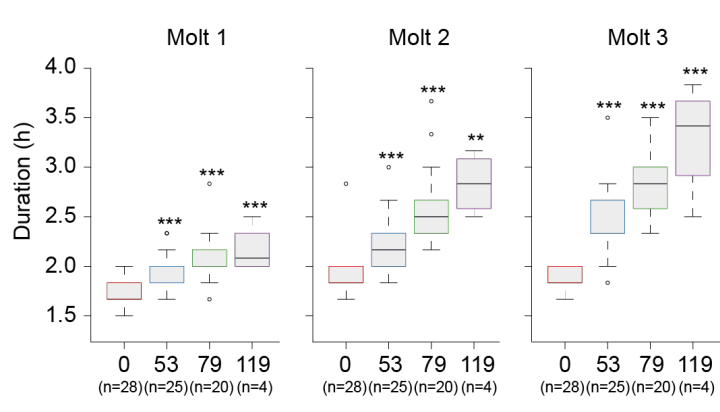

C
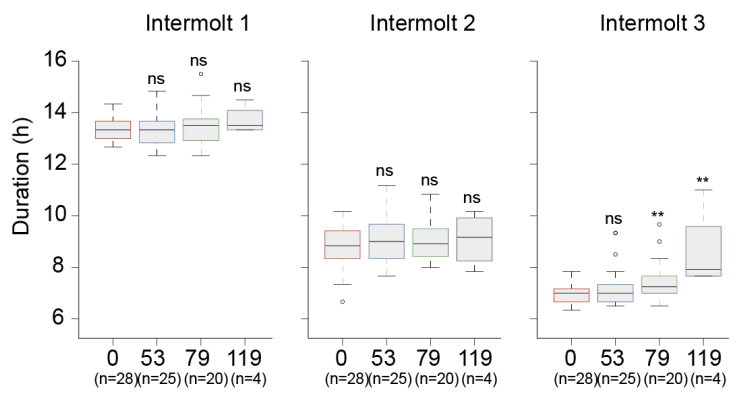

Auxin concentration ( $\mathrm{nM}$ )

Figure 3: GRH-1 depletion extends molt duration in a dose-dependent manner.

A, Quantification of the percentage of aid::grh-1 animals constitutively expressing luciferase (HW2434) that enter indicated molts after hatching into increasing concentrations of auxin as indicated. Molts in single animals grown at $20^{\circ} \mathrm{C}$ were determined as in Figure $\mathbf{S 1}$. For concentrations up to $250 \mu \mathrm{M}$, similar results as for $400 \mathrm{nM}$ were observed (Figure S3).

B, Boxplot showing the duration of $\mathrm{M} 1, \mathrm{M} 2$ and $\mathrm{M} 3$ of animals treated with indicated concentrations of auxin. Animals that revealed the phenotype at M1, M2 or M3 in (A) were excluded. Significant differences relative to $0 \mathrm{nM}$ auxin are indicated. P-values were determined by Wilcoxon test. ns: not significant, ${ }^{*} p<0.05,{ }^{* *} p<0.01,{ }^{* * *} p<0.001$

\section{Loss of GRH-1 results in fragile cuticles prone to rupturing}

When we placed grh-1::aid animals on auxin-containing plates, they failed to shed the cuticle (ecdyse). To observe the molting process in greater detail, we used time-lapse DIC imaging to observe L1 animals transferred to an agar pad in a drop of M9 buffer, which allowed them to move. We could readily identify loosened cuticles at the tip of the head in wild-type and GRH-1-depleted animal (Figure 4A,B). Next, animals made spontaneous back-and-forth movements and the pharynx contracted rapidly (Supplemental Movies 1 \& 2), after which wild-type animals shed the cuticle (ecdysed) (Figure 4A, B). By contrast, in GRH-1-depleted animals, the cuticle became even looser and more inflated in the head region

(Figure 4C). Vesicles appeared in the cavity underneath the loosened cuticle (Figure 4C). Finally, the cuticle broke in the head region and the underlying tissue was extruded (Figure 4C).

These observations likely explain the striking increase and later drop in signal following the aberrant molt observed in the luciferase assays for GRH-1-depleted animals (Fig. S1, Fig. 1). Indeed, even under conditions where more modest GRH-1 depletion remains compatible with survival, we can observe 
evidence of impaired cuticular integrity: Whereas lack of luciferin ingestion generates a low luminescence signal in molting wild-type animals, the signal increased in GRH-1-depleted animals during this time (Figure 4D).

We conclude GRH-1 is required for proper cuticle formation and timely ecdysis. In its absence, the duration of the molt becomes extended while a more fragile cuticle than typical of wild-type animals is formed. We note that although rupturing is lethal, animals also fail to complete development when grown on plate, a condition under which most animals do not burst but nonetheless fail to ecdyse (data not shown).

A
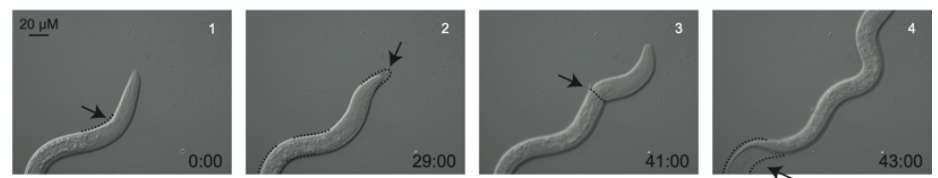

B
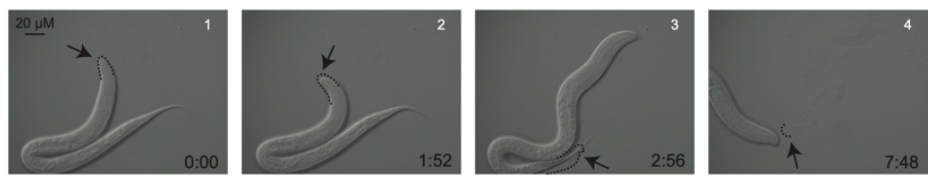

C
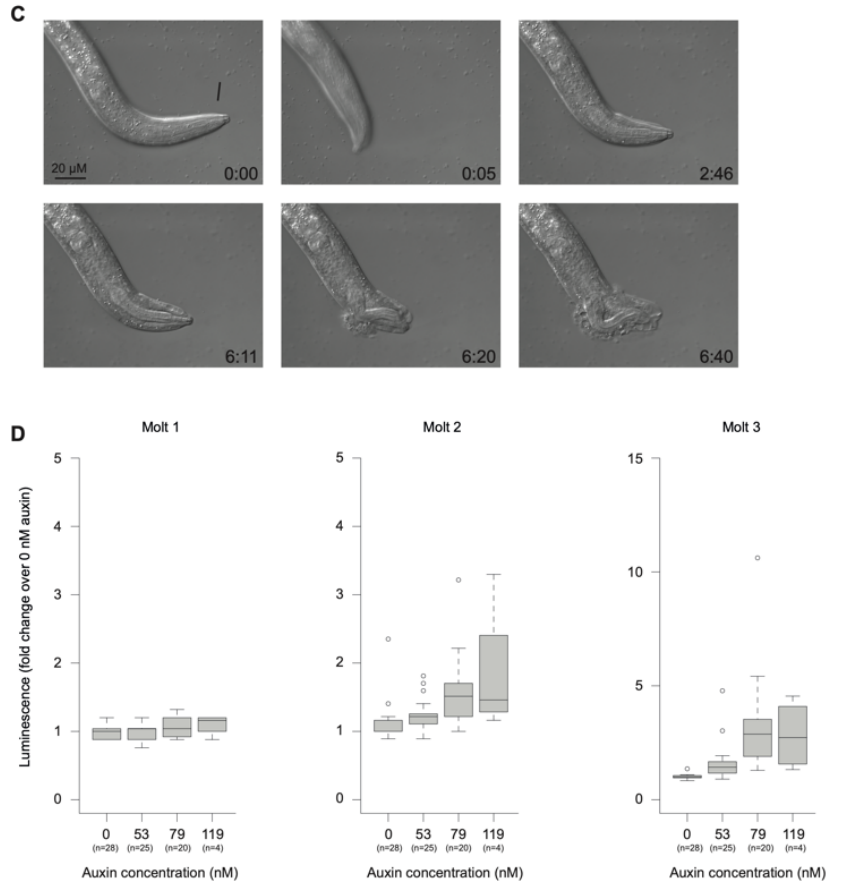

Figure 4: GRH-1 is required for cuticular integrity and normal ecdysis

A, B, Image sequence of N2 wild-type animals during M1. Lethargic animals were transferred to an agar-pad and observed by DIC at $63 x$ magnification and imaged every $20 \mathrm{~s}$ (A) or every $4 \mathrm{~s}$ (B) respectively. Selected images with time stamps are shown. Dashed lines indicate cuticle boundaries detached from the body. As reported previously (Singh and Sulston, 1978), two different sequences of events were observed: the pharyngeal lining is removed prior 
to ecdysis (A), or the pharyngeal lining is expelled after crawling out of the cuticle (B). Arrows indicate specific features of the molt. In (A): 1. Loosened cuticle, 2. Detachment of pharyngeal lining, 3. Crawling out of cuticle, 4. Final crawling out of cuticle. In (B): 1. Loosened cuticle, 2. Back-and-forth movements, 3. Crawling out of cuticle with pharyngeal lining still attached, 4. Pharyngeal lining expelled.

C, Image sequence of an L1 synchronized aid::grh-1 animal (HW2418) plated on $250 \mu \mathrm{M}$ auxin-containing plates, grown at $20^{\circ} \mathrm{C}$. A lethargic animal was transferred to an agar-pad containing microscopy slide and images were collected every $1 \mathrm{sec}$, using DIC, 100x magnification. Selected images of Supplemental Movie 1 are shown. Time stamp (min:sec) is indicated. Arrows indicate phenotypic features: loosening of the cuticle (0:00); back-and-forth movements (0:05); inflation of the cuticle (2:46); vesicles underneath loosened cuticle (6:11); rupturing of the cuticle $(6: 20,6: 40)$.

D, Luminescence during molt was quantified for aid::grh-1 animals (HW2418) grown in the presence of the indicated auxin concentrations.

\section{GRH-1 protein levels oscillate and peak shortly before molt entry}

Since GRH-1 is required repetitively, for proper execution of each of the four molts, and its mRNA levels oscillate (Hendriks et al., 2014; Meeuse et al., 2020), we wondered whether GRH-1 protein accumulation is also rhythmic. To test this, we examined a GRH-1::GFP fusion protein expressed from the endogenous grh-1 locus. We observed the first detectable signal in elongating embryos (Figure S6), i.e., at the time when oscillatory gene expression initiates (Meeuse et al., 2020). In larvae, GFP signal accumulated in various tissues, including seam cells, vulva precursor cells, non-seam hypodermal cells, rectum cells, socket cells, and pharyngeal cells (Figure S7). Finally, in adults, which lack oscillatory gene expression (Meeuse et al., 2020), GFP::GRH-1 levels were greatly diminished or altogether absent in various tissues

(Figure S8).

To study the larval dynamics of grh-1 expression in more detail and relate it to developmental progression, we performed time-lapse microscopy of single animals grown in micro-chambers, acquiring fluorescence and bright-field images in parallel and with high temporal resolution as described previously (Meeuse et al., 2020). We observed rhythmic GRH-1 accumulation with a peak before molt entry (Figure 5). Thus, the temporal patterns and dynamics suggest a model whereby rhythmic changes in GRH-1 protein levels may generate rhythmic GRH-1 activity. 


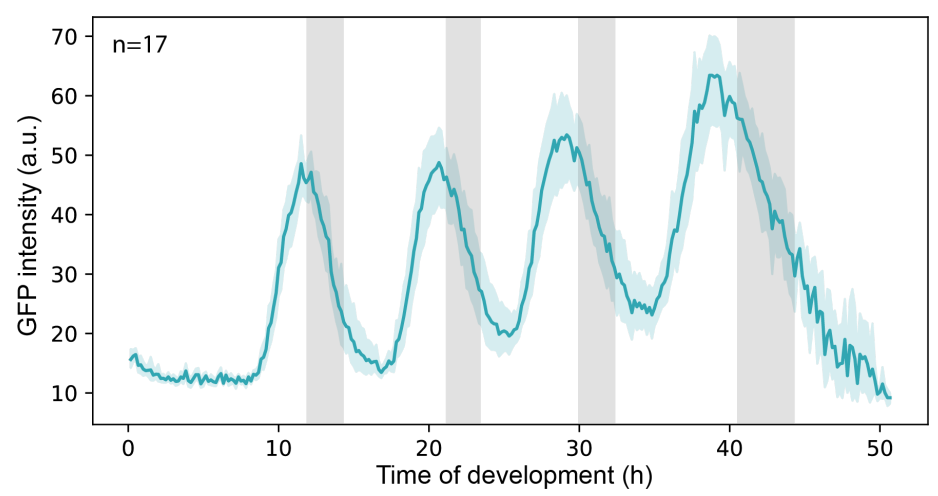

Figure 5: GRH-1 protein accumulates rhythmically before each molt

Time-lapse imaging of animals expressing endogenously tagged $g f p:: g r h-1$. Average +/- 95\% confidence interval (cyan shading) are shown; gray shading indicates average time of molt.

\section{Molting requires oscillatory GRH-1 activity}

To test further whether the activity of GRH-1 was indeed rhythmic, we performed perturbation at different times during a larval stage, to assess whether we could observe a phase-specific response. We initiated GRH-1 degradation at variable times in L2 by adding auxin and monitored developmental progression using the luciferase assay. Plotting luminescence traces by the time when the animals entered molt 2 in a heatmap (Figure 6A,B) revealed a striking cutoff on the onset of the phenotype: addition of auxin up to 3 hours before the M2 was sufficient for phenotypic onset at M2 exit (Figure 6B,C, green). However, if animals received auxin later than this, i.e., within 3 hours from $M 2$ entry or during $M 2$, they progressed through M2 and instead exhibited the phenotype at M3 exit (Figure 6B,C, blue). We observed analogous outcomes when auxin was added in L3 (Figure S9). This period of auxin resistance is not explained by slow GRH-1 depletion kinetics since auxin depletes GRH-1 effectively within < 1h (Figure 6D). Hence, a period of time exists in each larval stage during which GRH-1 is dispensable for molt completion. In other words, and as we had hypothesized, GRH-1 exhibits rhythmic activity during larval development. 
A

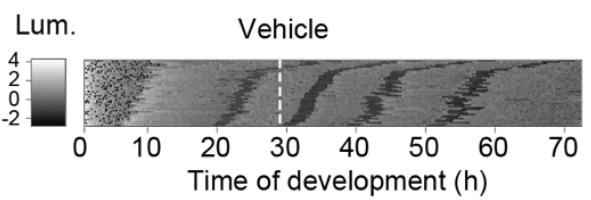

B

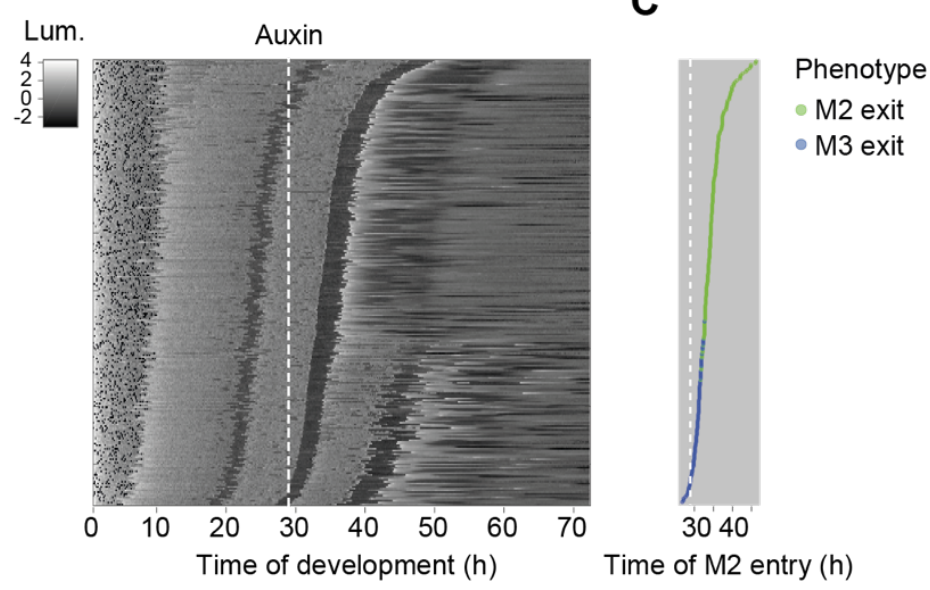

D

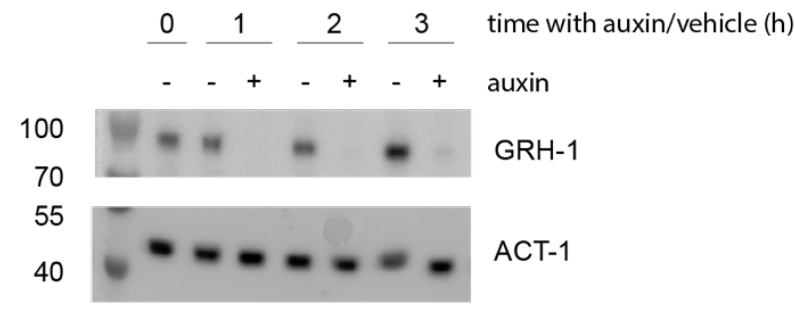

$(\mathrm{kDa})$

Figure 6: GRH-1 functions prior to the molt

A,B Heatmap showing trend-correct luminescence (Lum) of grh-1::aid animals (HW2434) treated with vehicle (0.25\% ethanol (A), or $250 \mu \mathrm{M}$ auxin (B) at 29 hours after plating (white dashed line). Black intensities reflect low luminescence during lethargus (molt). Embryos of various stages were plated to obtain an asynchronously hatching population. Luminescence traces are sorted by entry into molt 2 (M2) such that traces of early hatched animals are at the bottom and those of late hatched animals are at the top.

C, Plot of phenotype onset over time of auxin application relative to entry into molt 2 (M2 entry). Dots represent individual animals from (B), colored according to whether the last observed molt in the luminescence trace was $\mathrm{M} 2$ (M2 exit phenotype; green) or M3 (M3 exit phenotype; blue).

D, Western blot revealing rapid GRH-1 depletion in the grh-1::aid strain (HW2434) upon addition of $250 \mu \mathrm{M}$ auxin. A synchronized culture of animals was grown in liquid at $20^{\circ} \mathrm{C}$. After $21 \mathrm{~h}$ (denoted $\mathrm{t}=0 \mathrm{~h}$ in the figure), the culture was split in two and either auxin or vehicle were added as indicated. Cultures were sampled hourly and protein lysates probed by Western blotting using anti-GRH-1 and anti-actin antibodies as indicated. 


\section{Discussion}

Although molting is a fundamental feature of nematode development, little is known about the gene regulatory networks that control it. This is particularly true of the clock-type mechanisms thought to facilitate the regular occurrence of molts, and synchronization of the various processes that they encompasse. Considering that molting involves coordinated expression of tens or hundreds of extracellular matrix proteins, notably collagens, and their modifying enzymes, notably proteases and their inhibitors, transcription factors can be assumed to play important roles, but their identity and functions are not well established.

Here, we have identified six transcription factors with roles in molting and, potentially, oscillatory gene expression. Validating our approach, we identified NHR-23/Rora, arguably the best characterized molting transcription factor, whose depletion was previously shown to cause a failure of ecdysis and larval arrest (Frand et al., 2005; Gissendanner et al., 2004; Johnson et al., 2021; Kostrouchova et al., 1998; Kostrouchova et al., 2001; Kouns et al., 2011) and a role in regulating collagens and hedgehog-related genes (Kouns et al., 2011).

NHR-23 is orthologous to DHR3/Nr1f1, an ecdysone-controlled fly transcription factor important for metamorphosis (Kostrouchova 1998). In metamorphosis, DHR3 activates FTZ-F1 (Ou and King-Jones, 2013), the orthologue of a second one of our screen hits, NHR-25/Nr5a. Based on this orthology, and the finding that NHR-25 depletion impairs ecdysis, albeit more weakly and only in later molts than that of NHR-23, it was previously speculated that a regulatory interaction between the two proteins might be conserved in C. elegans and contribute to the molting process (Gissendanner et al., 2004; Gissendanner and Sluder, 2000). However, this notion has remained controversial and seems not supported by current evidence (Kostrouchova et al., 2001), suggesting that further effort will be needed to understand NHR-25 function in the molting cycle.

Elsewhere, we have reported a detailed characterization of a third screen hit, BLMP-1, which we found to modulate both molting timing and oscillatory gene expression (Hauser et al., 2021), possibly through its function as a pioneer transcription factor (Stec et al., 2021). Here, we have focused on characterizing another hit from our screen, GRH-1. Previously the least studied factor among our six leads, it is a member of the Grainyhead/LSF1 protein family that controls epithelial cell fates across animals (Sundararajan et al., 2020). Like NHR-23, it is repetively required for molting, but we additionally show that it acts during a specific window of each larval stage, before late intermolt. 
This window is consistent with, and presumably determined by, the rhythmic accumulation of GRH-1 protein, whose levels peak shortly before molt entry. We show that the failure to complete development observed in the screen is due to a cuticle and ecdysis defect: the onset of ecdysis is delayed and the newly formed cuticle is prone to rupturing during ecdysis, particularly in the larval head region. Consistent with the view that generation of the new cuticle is defective, rupturing happens specifically after the onset of ecdysis, with tissue extrusion occurring after the old (outer) cuticle has already visibly detached from the worm body. Indeed, the notion of impaired cuticle biogenesis is also consistent with cuticle defects in GRH-1-depleted C. elegans embryos, which do not undergo ecdysis (Venkatesan et al., 2003), although it remains possible that inappropriate proteolytic activities during ecdysis might additionally damage the newly formed cuticle.

Soft, thin and granular cuticles, prone to rupturing, are also a hallmark of Drosophila Grainyhead mutant animals (Bray and Kafatos, 1991; Nusslein-Volhard et al., 1984). In mammals, Grh/1 and Grh/3 are dynamically expressed in the epidermis (Joost et al., 2020; Joost et al., 2016) and control epidermal differentiation (Yu et al., 2006), wound healing, eye lid closure (Boglev et al., 2011). Hence, our findings agree with a fundamental, evolutionarily conserved role of Grainyhead proteins in epidermis and ECM development and remodeling.

Such parallels extend to additional screen hits, and we are particularly intrigued by the apparent similarites between $C$. elegans molting and the mammalian hair follicle cycle. This process of rhythmic homeostatic skin regeneration is controlled by a poorly characterized "hair follicle clock", modulated by circadian rhythms (Paus and Foitzik, 2004; Plikus et al., 2015). Mutations in the mouse orthologues of blmp-1 and $n h r-23, \operatorname{Prdm} 1$ and Rora, respectively, impair the proper execution of hair follicle cycles (Magnusdottir et al., 2007; Steinmayr et al., 1998; Telerman et al., 2017), while grh/1 ablation causes hair coat defects related to hair anchoring and potentially other processes (Wilanowski et al., 2008)

In summary, we propose that in this study we have identified and characterized a number of factors linked to a molting cycle oscillator that may have a functional correspondence in other animals, and that their further dissection provides a path to a molecular-mechanistic and systems understanding of gene expression oscillations in C. elegans. Additionally, we propose that $C$. elegans molting could serve as a powerful, genetically accessible, model of animal skin development and regeneration. 


\section{Methods}

\section{Transgenic animals}

HW1939: EG6699, xeSi296 [eft-3p::Iuc::gfp::unc-54 3'UTR, unc-119(+)] II (Meeuse et al., 2020)

HW1949: EG8080, xeSi301 [eft-3p::Iuc::gfp::unc-54 3'UTR, unc-119(+)] III (this study)

rde-1(ne219) V (Tabara et al., 1999)

HW2150: EG6699, xeSi296 [eft-3p::Iuc::gfp::unc-54 3'UTR, unc-119(+)] II; rde-1(ne219) V (this study)

CA1200: ieSi57 [eft-3p::TIR1::mRuby::unc-54 3'UTR, cb-unc-119(+)] II (Zhang et al., 2015)

HW1984: ieSi57 [eft-3p::TIR1::mRuby::unc-54 3'UTR, cb-unc-119(+)] II; EG8080, xeSi301 [eft3p::luc::gfp::unc-54 3'UTR, unc-119(+)] III (this study)

HW2079: EG8080, xeSi376 [eft-3p::TIR1::mRuby::unc-54 3'UTR, cb-unc-119(+)] III (this study)

HW2418: grh-1(xe135(grh-1::degron::3xFLAG)) I; EG8080, xeSi376 [eft-3p::TIR1::mRuby::unc-54 3'UTR, cb-unc-119(+)] III (this study)

HW2434: grh-1(xe135(grh-1::degron::3xFLAG)) I; EG6699, xeSi296 [eft-3p::luc::gfp::unc-54 3'UTR, unc119(+)] II; EG8080, xeSi376 [eft-3p::TIR1::mRuby::unc-54 3'UTR, cb-unc-119(+)] III (this study)

HW2493: grh-1(xe146) I; hT2

HW2603: grh-1(syb616(grh-1::GFP::3xFLAG)) I (PHX616, three times backcrossed) (this study; custommade by SunyBiotech)

\section{Generation of transgenic animals}

Endogenous aid::3xflag tagging grh-1 and generation of grh-1(0) mutants by CRISPR/Cas9-mediated editing was performed using the previously published $d p y-10$ (cn64) co-conversion protocol (Arribere et al., 2014). For the sgRNA plasmid, we inserted the sgRNA sequence (5' agaggtttactctcatgagt $\left.3^{\prime}\right)$ into Notldigested pIK198 (Katic et al., 2015) by Gibson assembly (Gibson et al., 2009).

For degron-3XFLAG tagging, we used hybridized MM116 (5' AATTGCAAATCTAAATGTTTagaggtttactctcatgagtGTTTAAGAGCTATGCTGGAA $\left.\quad 3^{\prime}\right)$ and MM117 (5' TTCCAGCATAGCTCTTAAACactcatgagagtaaacctctAAACATTTAGATTTGCAATT 3'). A degron-linker-3XFLAGlinker cassette was synthesized as gBlocks ${ }^{\circledR}$ Gene Fragments (Integrated DNA Technologies) with 50 bp 
homology arms to the grh-1 locus before the stopcodon: 5' CCACGTTAATCGAGGTGGCTCCCACCAATCCAAACTCGTATTCCAACTCAATGCCTAAAGATCCAGCCAAACCTCCG GCCAAGGCACAAGTTGTGGGATGGCCACCGGTGAGATCATACCGGAAGAACGTGATGGTTTCCTGCCAAAAATCA AGCGGTGGCCCGGAGGCGGCGGCGTTCGTGAAGAGTACCTCAGGCGGCTCGGGTGGTACTGGCGGCAGCGACT ACAAAGACCATGACGGTGATTATAAAGATCATGACATCGATTACAAGGATGACGATGACAAGAGTACTAGCGGTG GCAGTGGAGGTACCGGCGGAAGCTGAGAGTAAACCTCTTTAGGTTCTTGTCTTAATTCTCTTAAAGGAGGACT 3’. Wildtype animals were injected with $10 \mathrm{ng} / \mu \mathrm{L}$ gBlock, $100 \mathrm{ng} / \mu \mathrm{L}$ sgRNA plasmid, $20 \mathrm{ng} / \mu \mathrm{L}$ AF-ZF-827

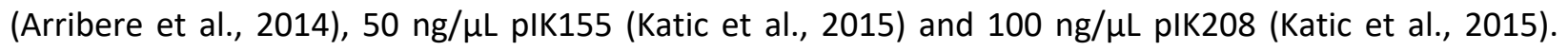
Genome editing was confirmed by sequencing.

For grh-1(0), we used hybrized MM116 and MM117, and hybrized MM118 (5' AATTGCAAATCTAAATGTTTGCGAAAATGCAAACATTAGAGTTTAAGAGCTATGCTGGAA 3') and MM119 (5' TTCCAGCATAGCTCTTAAACTCTAATGTTTGCATTTTCGCAAACATTTAGATTTGCAATT 3') to delete the coding region of $g r h-1$ and generate the $g r h-1$ (xe146) allele. Wildtype animals were injected with $100 \mathrm{ng} / \mu \mathrm{L}$ of each sgRNA plasmids, $19.2 \mathrm{ng} / \mu \mathrm{L}$ AF-ZF-827 (Arribere et al., 2014), $47.9 \mathrm{ng} / \mu \mathrm{L}$ plK155 (Katic et al., 2015) and $95.8 \mathrm{ng} / \mu \mathrm{L}$ plK208 (Katic et al., 2015). Genome editing was confirmed by sequencing. grh-1(xe146) homozygous animals could not be maintained and the grh-1(xe146) allele was balanced with hT2.

\section{Luciferase assays}

Luciferase assays were performed as described before (Meeuse et al., 2020). In short, single embryos, expressing luciferase from a constitutive and ubiquitous promoter (xeSi296 transgene) were placed in a 384-well plate (Berthold Technologies, 32505) by pipetting, and left to develop until adulthood in 90 uL SBasal medium containing E. coli OP50 $\left(\mathrm{OD}_{600}=0.9\right)$ and $100 \mu \mathrm{M}$ Firefly D-Luciferin (p.j.k., 102111). Luminescence was measured using a luminometer (Berthold Technologies, Centro XS3 LB 960) every 10 minutes for $0.5 \mathrm{sec}$ for 72 hours in a temperature controlled incubator set to 20 degrees.

For auxin experiments, a 400x stock solution of 3-indoleacetic acid (auxin, Sigma Aldrich, 12886) in 100\% ethanol was prepared. The stock solution was 400x diluted in the culture medium at the start each experiment or at specific time points and in concentrations as indicated.

Luminescence data was analyzed using an automated algorithm to detect the hatch and the molts in MATLAB with the possibility to annotate molts manually, as described before (Meeuse et al., 2020). Completion of molts was scored by the presence of a drop in luminescence, followed by a period of stable and low luminescence and subsequent rise in luminescence. 


\section{RNAi screen}

To knock-down 92 'oscillating' TRANSCRIPTION FACTOR, we used the RNAi feeding method. E. coli HT115 bacteria carrying RNAi plasmids were obtained from either of the two libraries (Ahringer library (Fraser et al., 2000; Kamath et al., 2003), Vidal library (Rual et al., 2004)) or cloned (see generation of RNAi vectors). Luciferase assays were performed as described above with the following adaptations: RNAi bacteria were grown in $5 \mathrm{~mL}$ auto-induction medium (2 mM MgSO4, $3.3 \mathrm{~g} / \mathrm{L}$ (NH4)2SO4, $6.8 \mathrm{~g} / \mathrm{LKH} 2 \mathrm{PO} 4,7.1 \mathrm{~g} / \mathrm{L}$ Na2HPO4, 5 g/L glycerol, $0.5 \mathrm{~g} / \mathrm{L}$ glucose, $2 \mathrm{~g} / \mathrm{L} \alpha$-lactose, $100 \mathrm{ug} / \mathrm{mL}$ Amp in ZY medium (10 g/L tryptone, $5 \mathrm{~g} / \mathrm{L}$ yeast extract)) at $37^{\circ} \mathrm{C}$. Bacteria were diluted in S-Basal medium (OD600 $\left.=0.45\right)$, with $100 \mathrm{uM}$ Firefly D-luciferin (p.j.k., 102111) and 100 ug/mL Ampicillin.

We used HW1939 animals that express the xeSi296 transgene. As a control strain, we used HW2150 animals expressing xeSi296 in an rde-1(ne219) (Tabara et al., 1999) background, which are RNAi deficient. For each RNAi condition we used 2 adjacent columns in the 384-wells plate, i.e. 32 wells with 90 uL culture medium each. To avoid plate effects, the first 8 wells of the first column and the last 8 wells of the second column of the same RNAi condition were filled with an HW1939 animal and the remaining wells with an HW2150 animal.

To identify mutants, we inspected the heatmaps with trend-corrected luminescence (Olmedo et al., 2015) for aberrant duration or number of molts and intermolts.

\section{Generation of RNAi vectors}

For clones that were not available in the Ahringer or Vidal libraries, cDNA or genomic DNA was PCR amplified using the following primers:

\begin{tabular}{|l|l|l|l|l|}
\hline locus & $\begin{array}{l}\text { vector } \\
\text { transformed }\end{array}$ & insert & Primer & Primer sequence \\
\hline nhr-5 & pMM012_R & Y73F8A.21a cDNA & $\begin{array}{l}\text { MM070 } \\
\text { MM071 }\end{array}$ & $\begin{array}{l}\text { ccaccggttccatggctagcTTCTGGCGGTAACAGTTCAA } \\
\text { ttgatatcgaattcctgcagGATGTGAGTATGGAATATTCGG }\end{array}$ \\
\hline dmd-8 & pMM013_R & T22H9.4 cDNA & MM077 & $\begin{array}{l}\text { ccaccggttccatggctagcCCCTGTCATCTTCTTCAAATGC } \\
\text { ttgatatcgaattcctgcagGTTTCAGCGCAGCTAATTGC }\end{array}$ \\
\hline bcl-11 & pMM014_R & F13H6.1a cDNA & $\begin{array}{l}\text { MM078 } \\
\text { ccaccggttccatggctagcAATAGAAACGTCTTCGGCGG } \\
\text { ttgatatcgaattcctgcagTTAACGGTTGGTGTGACTGC }\end{array}$ \\
\hline ztf-16 & pMM015_R & K03C7.2b cDNA & $\begin{array}{l}\text { MM080 } \\
\text { MM081 }\end{array}$ & $\begin{array}{l}\text { ccaccggttccatggctagcGATTTGCTACGATCACCCAT } \\
\text { ttgatatcgaattcctgcagGGCCTTGATTGGAGAAAGTG }\end{array}$ \\
\hline
\end{tabular}


bioRxiv preprint doi: https://doi.org/10.1101/2022.03.01.482504; this version posted March 4, 2022. The copyright holder for this preprint (which was not certified by peer review) is the author/funder, who has granted bioRxiv a license to display the preprint in perpetuity. It is made available under aCC-BY-NC 4.0 International license.

\begin{tabular}{|c|c|c|c|c|}
\hline sem-2 & pMM017_R & C32E12.5.1 CDNA & $\begin{array}{l}\text { MM084 } \\
\text { MM085 }\end{array}$ & $\begin{array}{l}\text { ccaccggttccatggctagcGATCTCCAAAAACCGCCCAA } \\
\text { ttgatatcgaattcctgcagTGCATCGCTCCATGGATAAT }\end{array}$ \\
\hline grh-1 & pMM018_R & Y48G8AR.1a cDNA & $\begin{array}{l}\text { MM086 } \\
\text { MM087 }\end{array}$ & $\begin{array}{l}\text { ccaccggttccatggctagcGAAGAAGTCCGACGGTGAAT } \\
\text { ttgatatcgaattcctgcagGAGTTTGGATTGGTGGGAGC }\end{array}$ \\
\hline dmd-9 & pMM019_R & Y67D8A.3 genomic DNA & $\begin{array}{l}\text { MM088 } \\
\text { MM089 }\end{array}$ & $\begin{array}{l}\text { ccaccggttccatggctagcCTTTGTTCCAGTTCAAACCAC } \\
\text { ttgatatcgaattcctgcagAGAGGGAAGGAACTGATAGAC }\end{array}$ \\
\hline M03D4.4b & pMM021_R & M03D4.4b cDNA & $\begin{array}{l}\text { MM092 } \\
\text { MM093 }\end{array}$ & $\begin{array}{l}\text { ccaccggttccatggctagcTCGGACACAGATTCATCACAAC } \\
\text { ttgatatcgaattcctgcagTCCGGTGTTGCTGTATTTGTC }\end{array}$ \\
\hline C08G9.2 & pMM022_R & C08G9.2 cDNA & $\begin{array}{l}\text { MM094 } \\
\text { MM095 }\end{array}$ & $\begin{array}{l}\text { ccaccggttccatggctagcTACCGGCAAGTGTACCAAAT } \\
\text { ttgatatcgaattcctgcagACCTTCACATGGATCTACACAA }\end{array}$ \\
\hline ets-4 & pMM024_R & F22A3.1a cDNA & $\begin{array}{l}\text { MM098 } \\
\text { MM099 }\end{array}$ & $\begin{array}{l}\text { ccaccggttccatggctagcATGCAATCTTCCAATCCAACC } \\
\text { ttgatatcgaattcctgcagAGGCAGGAATTTGTACACCA }\end{array}$ \\
\hline ztf-14 & pMM025_R & M163.2 genomic DNA & $\begin{array}{l}\text { MM102 } \\
\text { MM103 }\end{array}$ & $\begin{array}{l}\text { ccaccggttccatggctagcGCCGTCCCTGCATAACTACTC } \\
\text { ttgatatcgaattcctgcagAGAGAAGTGAGTTGCGGGAG }\end{array}$ \\
\hline ztf-29 & pMM026_R & Y66D12A.12 cDNA & $\begin{array}{l}\text { MM104 } \\
\text { MM105 }\end{array}$ & $\begin{array}{l}\text { ccaccggttccatggctagcCGTCACCGGCTCAACTTCCA } \\
\text { ttgatatcgaattcctgcagCATGTTCTCCTCCTTTCGCTCT }\end{array}$ \\
\hline
\end{tabular}

PCR fragments were cloned into the RNAi feeding Pml1 and Sma1 digested L4440 vector (L4440 was a gift from Andrew Fire (Addgene plasmid \# 1654 ; http://n2t.net/addgene:1654 ; RRID:Addgene_1654)) using Gibson assembly (Gibson et al., 2009)) and transfected into E. coli HT115 bacteria.

\section{Phenotype imaging}

For imaging phenotypes, HW2418 worms were mounted on a $2 \%(\mathrm{w} / \mathrm{v})$ agarose pad with a drop of M9 buffer (42 $\mathrm{mM} \mathrm{Na}_{2} \mathrm{HPO}_{4}, 22 \mathrm{mM} \mathrm{KH}_{2} \mathrm{PO}_{4}, 86 \mathrm{mM} \mathrm{NaCl}, 1 \mathrm{mM} \mathrm{MgSO}$ ). grh-1::aid animals were imaged on an Axio Imager Z1 (Zeiss) microscope. We acquired Differential Interference Contrast (DIC) images using a 100x/1.4 oil immersion objective and a TL Halogen Lamp (3.00 Volt, 900 ms exposure). Images (1388x1040 pixels, $142.1 \mu \mathrm{m} \times 106.48 \mu \mathrm{m}$ pixel size, $12 \mathrm{Bit}$ ) were acquired every second from the moment that the cuticle became loose around the tip of the head until after the worm burst through the head, which took roughly 5 to 10 minutes. N2 animals were imaged on an Axio Imager Z2 (Zeiss) microscope. We acquired Differential Interference Contrast (DIC) images using a 63x/1.4 oil immersion objective) and a TL Vis-LED Lamp (5.74 Volt, 17 ms (Figure 4A) or 19 ms (Figure 4B) exposure). Images (1388x1040 pixels, $225.56 \mu \mathrm{m} \times 169.01 \mu \mathrm{m}$ pixel size, $12 \mathrm{Bit}$ ) were acquired at 20 seconds ((Figure $4 \mathrm{~A}$ ) or 4 seconds (Figure 
4B) intervals from the moment that the cuticle became loose around the tip of the head until the cuticle was shed.

\section{Single worm imaging}

To investigate the temporal expression pattern of endogenously tagged grh-1::gfp, strain HW2603 (grh1(syb616(grh-1::GFP::3xFLAG)) I) was observed by time lapse imaging using single worm imaging as previously described (Meeuse et al., 2020) with slight modifications. Briefly, an array of microchambers (Bringmann, 2011; Turek et al., 2015) was made of 4.5\% agarose in S-Basal medium (Stiernagle, 2005). OP50 bacteria were grown on agar plates, scraped off, and transferred to the chambers. Single eggs were placed on the chambers and flipped into a glass coverslip surrounded by a silicone insulator. Low melting agarose (3.5\%) was used to seal the edges of the array, which was subsequently mounted on a glass slide for imaging. We imaged animals using a 2x sCMOS camera model (T2) on an Axio Imager M2 (upright microsocope) CSU_W1 Yokogawa spinning disk microscope with a 20x air objective (NA=0.8). The $488 \mathrm{~nm}$ laser was set to $70 \%$, with $10 \mathrm{~ms}$ exposure and a binning of 2 . The brightfield and fluorescent images were taken in parallel using a motorized z-drive with a 2 - $\mu \mathrm{m}$ step size and 23 images per z-stack for a total duration of $60 \mathrm{~h}$ at $10 \mathrm{~min}$ time intervals in a $21^{\circ} \mathrm{C}$ room.

\section{Single worm imaging data analysis}

Brightfield images were segmented using a Convolutional Neural Network (CNN v2) (to be communicated elsewhere). Using a default threshold of 127 on the segmentation probability, GFP intensities were quantified on the segmented images using a previously published KNIME workflow (Meeuse et al., 2020). In short, worms are straightened and the GFP intensity of the worm is max projected to one pixel line for each time point. Background-subtracted mean GFP intensities are determined from $20 \%-80 \%$ of the anterior-posterior axis for each time point.To annotate molts, each image was visually inspected for molt entry and molt exit by scrolling through z-stack of the individual timepoints. The GFP intensities and lethargus data was plotted together in Python v3.9 using the Seaborn package.

\section{Acknowledgements}

We thank Iskra Katic and Lan Xu for help in generating transgenic strains, Anca Neagu for support in analyzing grh-1 mutant phenotypes, Yannick Hauser, Marit van der Does, Markus Rempfler, Benjamin 
Titze, Jan Eglinger and Laurent Gelman for help with imaging and image analysis, and Dimos Gaidatzis for help in analyzing luciferase screening data.

\section{Funding}

M.W.M.M. received support from a Boehringer Ingelheim Fonds PhD fellowship. S.N. from a Marie Sklodowska-Curie grant under the EU Horizon 2020 Research and Innovation Program (Grant agreement No. 842386). This work is part of a project that has received funding from the European Research Council (ERC) under the European Union's Horizon 2020 research and innovation programme (Grant agreement No. 741269, to H.G.). The FMI is core-funded by the Novartis Research Foundation.

\section{Author contributions}

K.B. imaged the N2 molt. S.N. performed GRH-1 time-lapse imaging and related data analysis. M.W.M.M performed the remaining experiments and analyzed the associated data. H.G. and M.W.M.M conceived the project and wrote the manuscript. 


\section{References}

Arribere, J.A., Bell, R.T., Fu, B.X., Artiles, K.L., Hartman, P.S., and Fire, A.Z. (2014). Efficient marker-free recovery of custom genetic modifications with CRISPR/Cas9 in Caenorhabditis elegans. Genetics 198, 837846.

Boglev, Y., Wilanowski, T., Caddy, J., Parekh, V., Auden, A., Darido, C., Hislop, N.R., Cangkrama, M., Ting, S.B., and Jane, S.M. (2011). The unique and cooperative roles of the Grainy head-like transcription factors in epidermal development reflect unexpected target gene specificity. Dev Biol 349, 512-522.

Bray, S.J., and Kafatos, F.C. (1991). Developmental function of Elf-1: an essential transcription factor during embryogenesis in Drosophila. Genes Dev 5, 1672-1683.

Bringmann, H. (2011). Agarose hydrogel microcompartments for imaging sleep- and wake-like behavior and nervous system development in Caenorhabditis elegans larvae. J Neurosci Methods 201, 78-88.

Cohen, J.D., Sparacio, A.P., Belfi, A.C., Forman-Rubinsky, R., Hall, D.H., Maul-Newby, H., Frand, A.R., and Sundaram, M.V. (2020). A multi-layered and dynamic apical extracellular matrix shapes the vulva lumen in Caenorhabditis elegans. Elife 9, e57874.

Cohen, J.D., and Sundaram, M.V. (2020). C. elegans Apical Extracellular Matrices Shape Epithelia. J Dev Biol 8.

Frand, A.R., Russel, S., and Ruvkun, G. (2005). Functional genomic analysis of C. elegans molting. PLoS Biol 3, e312.

Fraser, A.G., Kamath, R.S., Zipperlen, P., Martinez-Campos, M., Sohrmann, M., and Ahringer, J. (2000). Functional genomic analysis of C. elegans chromosome I by systematic RNA interference. Nature 408, 325330.

Gibson, D.G., Young, L., Chuang, R.-Y., Venter, J.C., Hutchison, C.A., and Smith, H.O. (2009). Enzymatic assembly of DNA molecules up to several hundred kilobases. Nature Methods 6, 343-345.

Gissendanner, C.R., Crossgrove, K., Kraus, K.A., Maina, C.V., and Sluder, A.E. (2004). Expression and function of conserved nuclear receptor genes in Caenorhabditis elegans. Dev Biol 266, 399-416.

Gissendanner, C.R., and Sluder, A.E. (2000). nhr-25, the Caenorhabditis elegans ortholog of ftz-f1, is required for epidermal and somatic gonad development. Dev Biol 221, 259-272.

Hauser, Y.P., Meeuse, M.W.M., Gaidatzis, D., and Großhans, H. (2021). The BLMP-1 transcription factor promotes oscillatory gene expression to achieve timely molting. bioRxiv, 2021.2007.2005.450828.

Hendriks, G.-J., Gaidatzis, D., Aeschimann, F., and Großhans, H. (2014). Extensive oscillatory gene expression during $C$. elegans larval development. Mol Cell 53, 380-392.

Johnson, L.C., Aguilera, J., Levenson, M.T., Rechtsteiner, A., Vo, A.A., Ragle, J.M., and Ward, J.D. (2021). Conditional depletion reveals temporal requirements for the oscillating transcription factor NHR23/NR1F1 in <em>C. elegans</em> larval progression. bioRxiv, 2021.2010.2027.465992.

Joost, S., Annusver, K., Jacob, T., Sun, X., Dalessandri, T., Sivan, U., Sequeira, I., Sandberg, R., and Kasper, M. (2020). The Molecular Anatomy of Mouse Skin during Hair Growth and Rest. Cell Stem Cell 26, 441457 e447.

Joost, S., Zeisel, A., Jacob, T., Sun, X., La Manno, G., Lonnerberg, P., Linnarsson, S., and Kasper, M. (2016). Single-Cell Transcriptomics Reveals that Differentiation and Spatial Signatures Shape Epidermal and Hair Follicle Heterogeneity. Cell Syst 3, 221-237 e229.

Kamath, R.S., Fraser, A.G., Dong, Y., Poulin, G., Durbin, R., Gotta, M., Kanapin, A., Le Bot, N., Moreno, S., Sohrmann, M., et al. (2003). Systematic functional analysis of the Caenorhabditis elegans genome using RNAi. Nature 421, 231-237.

Katic, I., Xu, L., and Ciosk, R. (2015). CRISPR/Cas9 Genome Editing in Caenorhabditis elegans: Evaluation of Templates for Homology-Mediated Repair and Knock-Ins by Homology-Independent DNA Repair. G3 (Bethesda) 5, 1649-1656. 
Kim, D.h., Grün, D., and van Oudenaarden, A. (2013). Dampening of expression oscillations by synchronous regulation of a microRNA and its target. Nat Genet 45, 1337-1344.

Kostrouchova, M., Krause, M., Kostrouch, Z., and Rall, J.E. (1998). CHR3: a Caenorhabditis elegans orphan nuclear hormone receptor required for proper epidermal development and molting. Development 125, 1617-1626.

Kostrouchova, M., Krause, M., Kostrouch, Z., and Rall, J.E. (2001). Nuclear hormone receptor CHR3 is a critical regulator of all four larval molts of the nematode Caenorhabditis elegans. Proc Natl Acad Sci U S A 98, 7360-7365.

Kouns, N.A., Nakielna, J., Behensky, F., Krause, M.W., Kostrouch, Z., and Kostrouchova, M. (2011). NHR23 dependent collagen and hedgehog-related genes required for molting. Biochem Biophys Res Commun 413, 515-520.

Magnusdottir, E., Kalachikov, S., Mizukoshi, K., Savitsky, D., Ishida-Yamamoto, A., Panteleyev, A.A., and Calame, K. (2007). Epidermal terminal differentiation depends on B lymphocyte-induced maturation protein-1. Proc Natl Acad Sci U S A 104, 14988-14993.

Meeuse, M.W.M., Hauser, Y.P., Hendriks, G.-J., Eglinger, J., Bogaarts, G., Tsiairis, C., and Großhans, H. (2020). Developmental function and state transitions of a gene expression oscillator in C. elegans. Mol Syst Biol 16, e9498.

Monsalve, G.C., and Frand, A.R. (2012). Toward a unified model of developmental timing: A "molting" approach. Worm 1, 221-230.

Nusslein-Volhard, C., Wieschaus, E., and Kluding, H. (1984). Mutations affecting the pattern of the larval cuticle inDrosophila melanogaster : I. Zygotic loci on the second chromosome. Wilehm Roux Arch Dev Biol 193, 267-282.

Olmedo, M., Geibel, M., Artal-Sanz, M., and Merrow, M. (2015). A High-Throughput Method for the Analysis of Larval Developmental Phenotypes in Caenorhabditis elegans. Genetics 201, 443-448.

Ou, Q., and King-Jones, K. (2013). What goes up must come down: transcription factors have their say in making ecdysone pulses. Curr Top Dev Biol 103, 35-71.

Paus, R., and Foitzik, K. (2004). In search of the "hair cycle clock": a guided tour. Differentiation 72, 489511.

Plikus, M.V., Van Spyk, E.N., Pham, K., Geyfman, M., Kumar, V., Takahashi, J.S., and Andersen, B. (2015). The circadian clock in skin: implications for adult stem cells, tissue regeneration, cancer, aging, and immunity. J Biol Rhythms 30, 163-182.

Rual, J.F., Ceron, J., Koreth, J., Hao, T., Nicot, A.S., Hirozane-Kishikawa, T., Vandenhaute, J., Orkin, S.H., Hill, D.E., van den Heuvel, S., et al. (2004). Toward improving Caenorhabditis elegans phenome mapping with an ORFeome-based RNAi library. Genome Res 14, 2162-2168.

Sandhu, A., Badal, D., Sheokand, R., Tyagi, S., and Singh, V. (2021). Specific collagens maintain the cuticle permeability barrier in Caenorhabditis elegans. Genetics 217.

Singh, R.N., and Sulston, J.E. (1978). Some observations on molting in Caenorhabditis elegans. Nematologica 24, 63-\&.

Stec, N., Doerfel, K., Hills-Muckey, K., Ettorre, V.M., Ercan, S., Keil, W., and Hammell, C.M. (2021). An Epigenetic Priming Mechanism Mediated by Nutrient Sensing Regulates Transcriptional Output during C. elegans Development. Curr Biol 31, 809-826 e806.

Steinmayr, M., Andre, E., Conquet, F., Rondi-Reig, L., Delhaye-Bouchaud, N., Auclair, N., Daniel, H., Crepel, F., Mariani, J., Sotelo, C., et al. (1998). staggerer phenotype in retinoid-related orphan receptor alphadeficient mice. Proc Natl Acad Sci U S A 95, 3960-3965.

Stiernagle, T. (2005). Maintenance of C. elegans (February 11, 2006), WormBook, ed. The C. elegans Research Community, WormBook, doi/10.1895/wormbook.1.101.1, http://www.wormbook.org. 
Sundararajan, V., Pang, Q.Y., Choolani, M., and Huang, R.Y. (2020). Spotlight on the Granules (GrainyheadLike Proteins) - From an Evolutionary Conserved Controller of Epithelial Trait to Pioneering the Chromatin Landscape. Front Mol Biosci 7, 213.

Tabara, H., Sarkissian, M., Kelly, W.G., Fleenor, J., Grishok, A., Timmons, L., Fire, A., and Mello, C.C. (1999). The rde-1 gene, RNA interference, and transposon silencing in C. elegans. Cell 99, 123-132.

Telerman, S.B., Rognoni, E., Sequeira, I., Pisco, A.O., Lichtenberger, B.M., Culley, O.J., Viswanathan, P., Driskell, R.R., and Watt, F.M. (2017). Dermal Blimp1 Acts Downstream of Epidermal TGFbeta and Wnt/beta-Catenin to Regulate Hair Follicle Formation and Growth. J Invest Dermatol 137, 2270-2281.

Tsiairis, C., and Großhans, H. (2021). Gene expression oscillations in C. elegans underlie a new developmental clock. Curr Top Dev Biol 144, 19-43.

Turek, M., Besseling, J., and Bringmann, H. (2015). Agarose Microchambers for Long-term Calcium Imaging of Caenorhabditis elegans. J Vis Exp, e52742.

Turek, M., and Bringmann, H. (2014). Gene expression changes of Caenorhabditis elegans larvae during molting and sleep-like lethargus. PLoS One 9, e113269.

Venkatesan, K., McManus, H.R., Mello, C.C., Smith, T.F., and Hansen, U. (2003). Functional conservation between members of an ancient duplicated transcription factor family, LSF/Grainyhead. Nucleic Acids Res 31, 4304-4316.

Wilanowski, T., Caddy, J., Ting, S.B., Hislop, N.R., Cerruti, L., Auden, A., Zhao, L.L., Asquith, S., Ellis, S., Sinclair, R., et al. (2008). Perturbed desmosomal cadherin expression in grainy head-like 1-null mice. EMBO J 27, 886-897.

Zhang, L., Ward, J.D., Cheng, Z., and Dernburg, A.F. (2015). The auxin-inducible degradation (AID) system enables versatile conditional protein depletion in C. elegans. Development 142, 4374-4384. 
bioRxiv preprint doi: https://doi.org/10.1101/2022.03.01.482504; this version posted March 4, 2022. The copyright holder for this preprint (which was not certified by peer review) is the author/funder, who has granted bioRxiv a license to display the preprint in perpetuity. It is made available under aCC-BY-NC 4.0 International license.

\section{Supplementary figures}
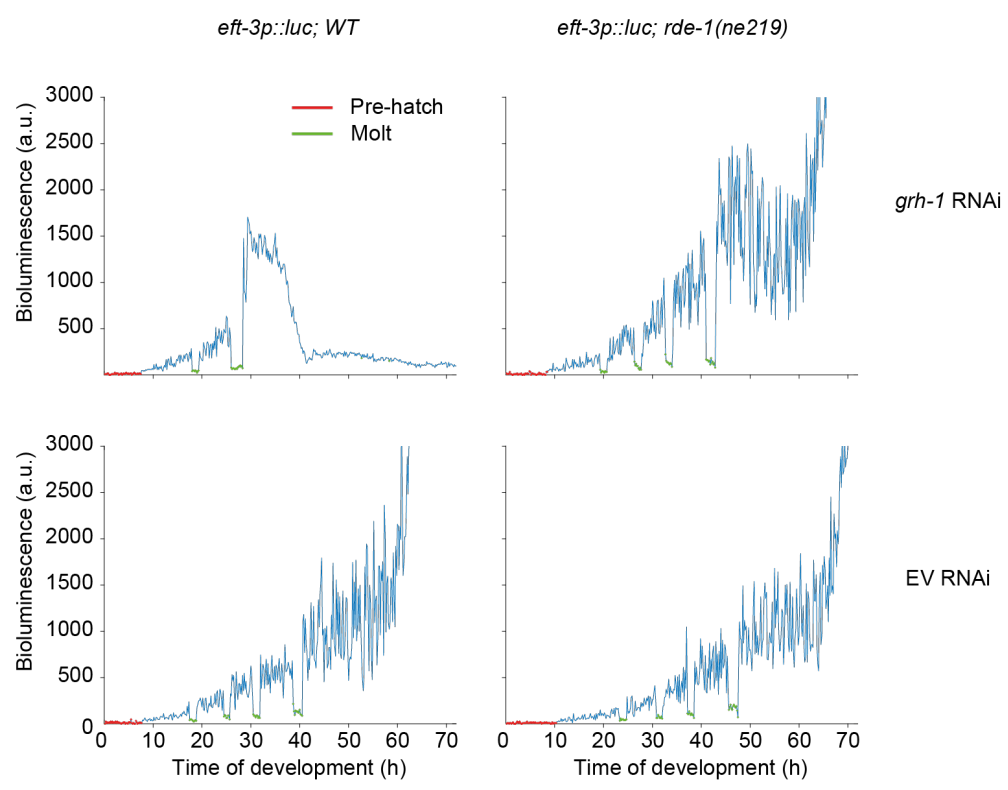

\section{Figure S1: Related to Figure 1 - grh-1 RNAi causes aberrant progression through development}

Representative raw luminescence trace of single animals grown at $20^{\circ} \mathrm{C}$ for 72 hours. Left column showing wildtype animals and right column RNAi-deficient animals (rde-1(ne219)). Animals in upper panel were grown in the presence of grh-1 RNAi and animals in the lower panel were grown in the presence of mock (empty vector) RNAi. Pre-hatch is indicated in red and molts in green. 
A

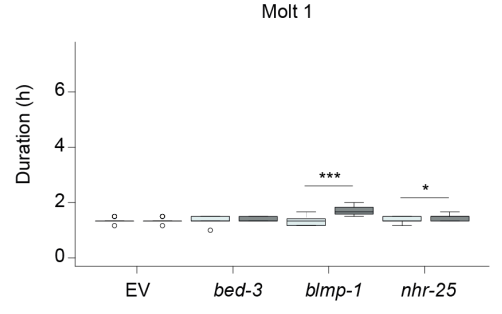

Molt 3

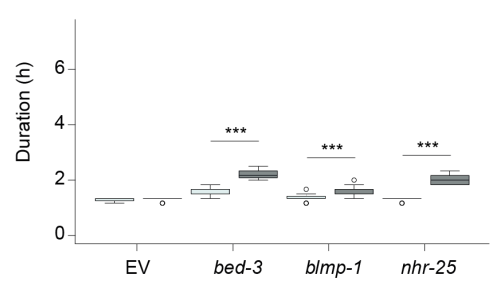

B

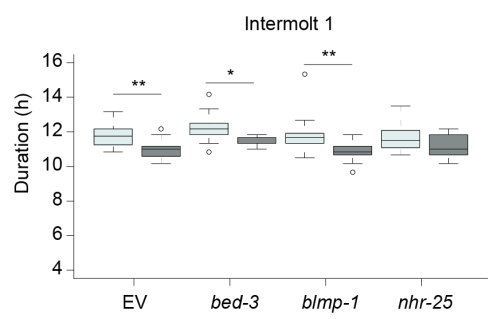

Intermolt 3

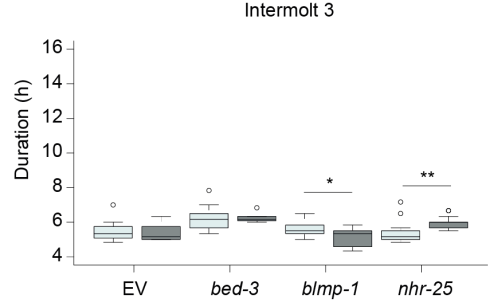

Molt 2
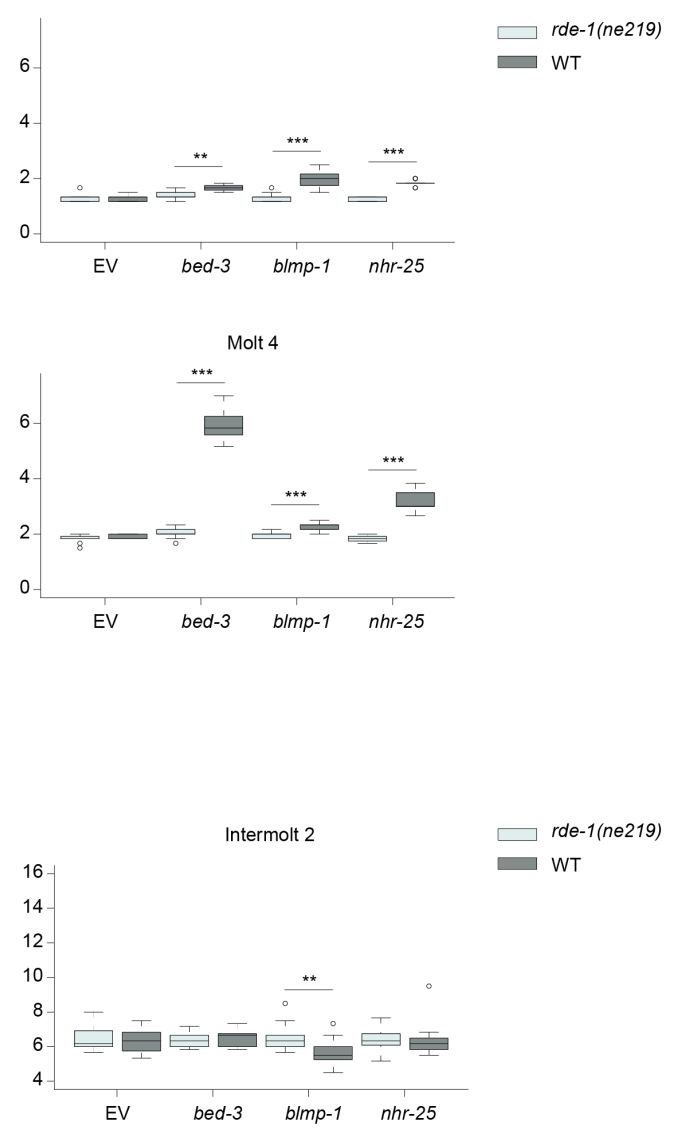

Intermolt 4

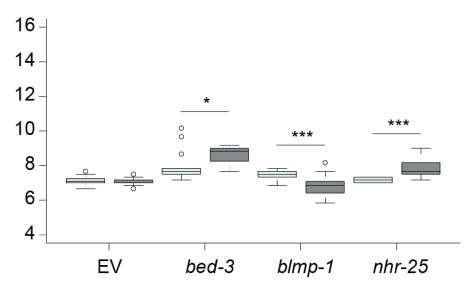

Figure S2: Related to Figure 1 - Quantification of molt and intermolt duration in screen hits that complete development

A, Quantification of molt durations in RNAi deficient (rde-1(ne219)) animals (light grey) grown in the presence of mock $(n=16)$, bed-3 $(n=14)$, blmp-1 $(n=16)$ or $n h r-25(n=15)$ RNAi, and wildtype animals (dark grey) grown in the presence of mock $(n=16)$, bed-3 $(n=8)$, blmp-1 $(n=16)$ or $n h r-25(n=13)$ RNAi. Significant differences relative to rde1(ne219) for each RNAi condition are indicated.

B, Quantification of intermolt durations in RNAi deficient (rde-1(ne219)) animals (light grey) grown in the presence of mock ( $n=16)$, bed-3 $(n=14)$, blmp-1 $(n=16)$ or $n h r-25(n=15) R N A i$, and wildtype animals (dark grey) grown in the presence of mock $(n=16)$, bed-3 $(n=8)$, blmp-1 $(n=16)$ or $n h r-25(n=13)$ RNAi. Significant differences relative to rde1(ne219) for each RNAi condition are indicated.

$* \mathrm{p}<0.05, * * \mathrm{p}<0.01, * * * \mathrm{p}<0.001$, Wilcoxon test. 
A
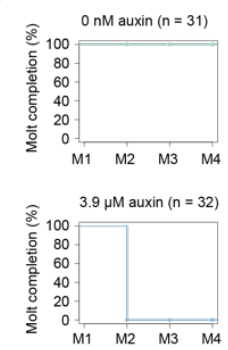

B

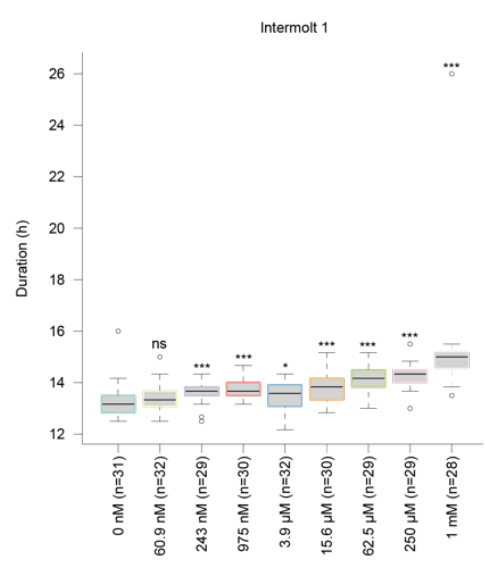

M1 $\quad$ M2 $\quad$ M3 $\quad$ M4
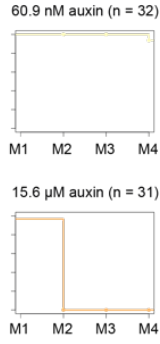
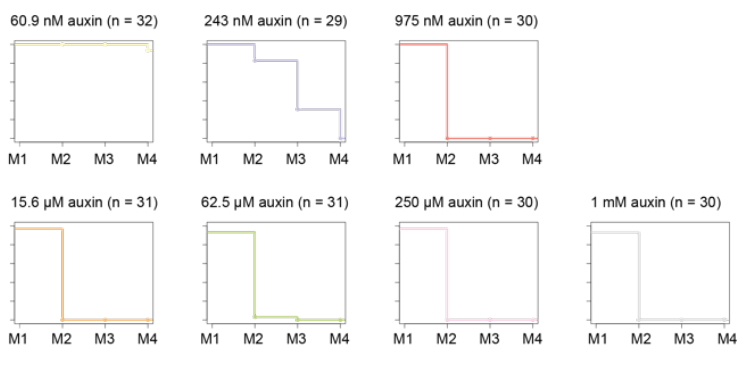

C

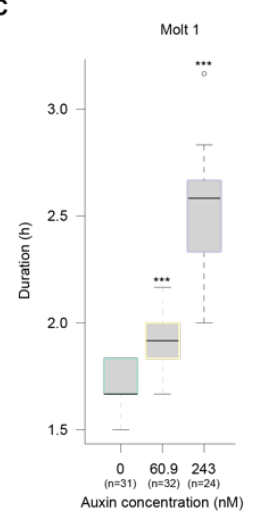

Figure S3: Related to Figure 3 - Intermolt is lengthened upon GRH-1 depletion at higher auxin concentrations

A, Quantification of the percentage of grh-1(xe135); eft-3p::Iuc; eft-3p::TIR1 animals (HW2434) that enter each of four molts molt upon increasing concentrations of auxin. Note that for some concentrations M1 does not start at $100 \%$, i.e. animals initiated I1 but M1 was not observed.

B, Boxplot showing the duration of the first intermolt of animals treated with indicated concentrations of auxin. Animals that failed to progress development beyond M1 (A). Significant differences relative to $0 \mathrm{nM}$ auxin are indicated.

C, Boxplot showing the duration of M1 of animals treated with indicated concentrations of auxin. Animals that failed to progress development beyond M1 were excluded (A). Significant differences relative to $0 \mathrm{nM}$ auxin are indicated.

P-values were determined by Wilcoxon test. ns: not significant ${ }^{*} p<0.05, * * p<0.01, * * * p<0.001$ 
A

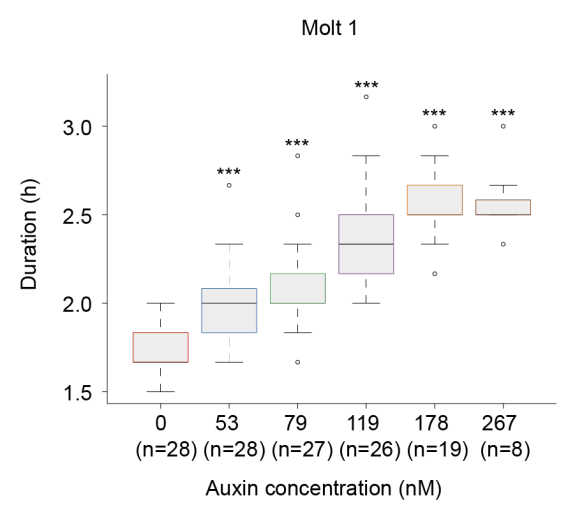

B

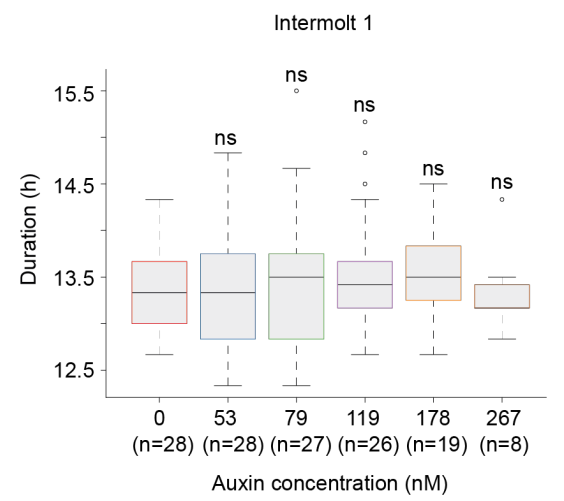

Auxin concentration (nM)
C

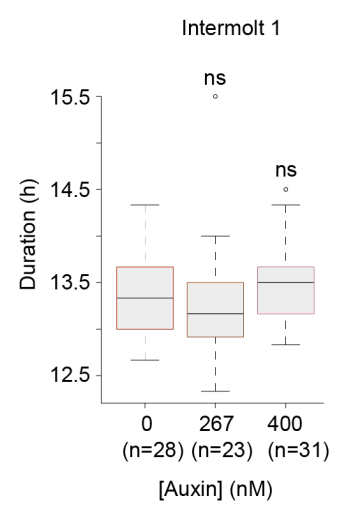

D

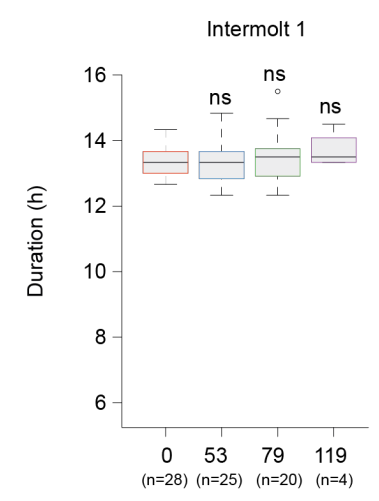

Intermolt 2

Intermolt 3
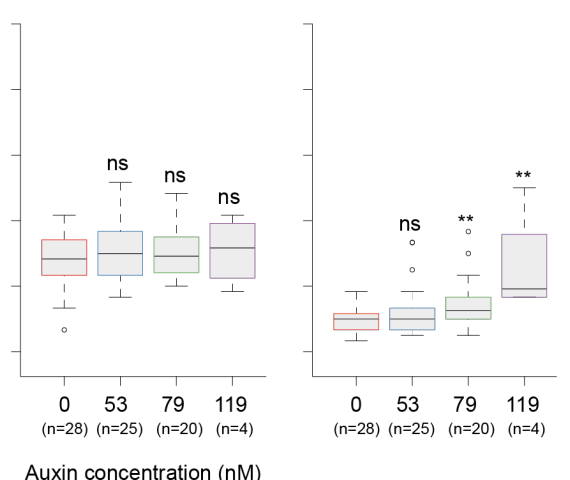

Figure S4: Related to Figure 3 - Low auxin concentrations strongly affect molt but not intermolt duration of aid::grh-1 animals

A, Boxplot showing the duration of the first molt of grh-1(xe135); eft-3p::luc; eft-3p::TIR1 animals (HW2434) treated with indicated concentrations of auxin. Animals that failed to progress development beyond M1 were excluded (Figure S3A). Significant differences relative to $0 \mathrm{nM}$ auxin are indicated.

B, Boxplot showing the duration of the first intermolt of animals treated with indicated concentrations of auxin. Animals that failed to progress development beyond M1 were excluded (Figure S3A). Significant differences relative to $0 \mathrm{nM}$ auxin are indicated.

C, Boxplot showing duration of first intermolt for vehicle-treated animals and animals treated with $267 \mathrm{nM}$ and 400 $\mathrm{nM}$ auxin (Figure S3A). For auxin-treated animals, only those that showed a phenotype at M1 were selected. Significant differences relative to $0 \mathrm{nM}$ auxin are indicated.

D, Boxplot showing the duration of 11,12 and 13 of animals treated with indicated concentrations of auxin. Animals that revealed the phenotype at M1, M2 or M3 were excluded (Figure S3A). Significant differences relative to $0 \mathrm{nM}$ auxin are indicated.

P-values were determined by Wilcoxon test. ns: not significant, * $p<0.05,{ }^{* *} p<0.01,{ }^{* * *} p<0.001$ 
A
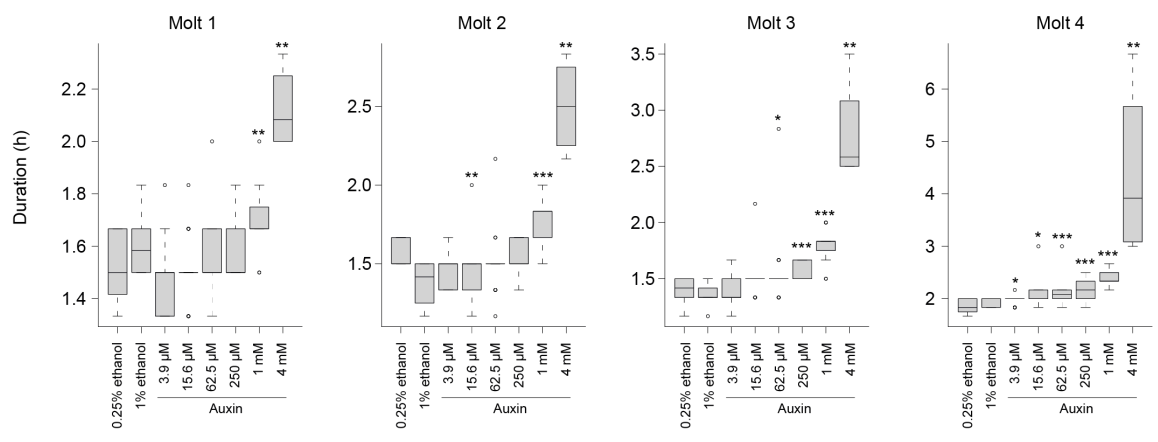

B
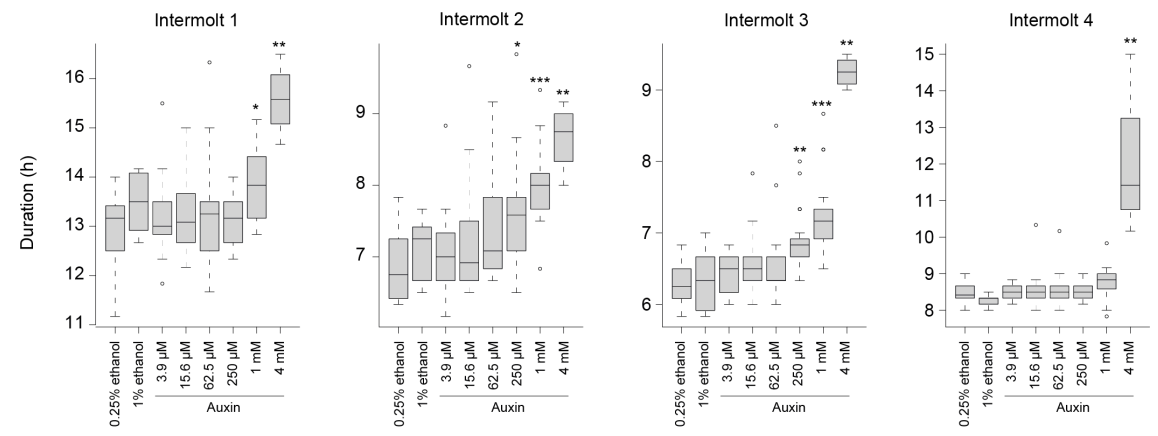

Figure S5: Related to Figure 3 - Effects of auxin in animal lacking a degron

A, Boxplots showing duration of each molt for indicated concentrations of auxin in eft-3p::TIR1; eft-3p::/uc animals (HW1984), which lack the aid tag on grh-1. Differences relative to $0.25 \%$ ethanol (0 nM auxin) for $3.9 \mu \mathrm{M}-1 \mathrm{mM}$ auxin and relative to $1 \%$ ethanol $\left(0 \mathrm{nM}\right.$ auxin) for $4 \mathrm{mM}$ auxin are indicated (ns: not significant, ${ }^{*} \mathrm{p}<0.05,{ }^{* *} \mathrm{p}<0.01$, *** $\mathrm{P}<0.001$, Wilcoxon test). Note that concentrations $\leq 250 \mu \mathrm{M}$ yield at most minor extensions of molt durations (compare Figure 3). At $4 \mathrm{mM}$ auxin, most animals fail to complete M4.

B, Boxplots showing duration of each intermolt for indicated concentrations of auxin in eft-3p::TIR1; eft-3p::Iuc animals (HW1984). Differences relative to $0.25 \%$ ethanol ( $0 \mathrm{nM}$ auxin) for $3.9 \mu \mathrm{M}-1 \mathrm{mM}$ auxin and relative to $1 \%$ ethanol ( $0 \mathrm{nM}$ auxin) for $4 \mathrm{mM}$ auxin are indicated (ns: not significant, ${ }^{*} p<0.05,{ }^{* *} p<0.01,{ }^{* * *} \mathrm{P}<0.001$, Wilcoxon test). Note that concentrations $\leq 250 \mu \mathrm{M}$ yield at most minor extensions of intermolt durations. 
bioRxiv preprint doi: https://doi.org/10.1101/2022.03.01.482504; this version posted March 4, 2022. The copyright holder for this preprin (which was not certified by peer review) is the author/funder, who has granted bioRxiv a license to display the preprint in perpetuity. It is made available under aCC-BY-NC 4.0 International license.
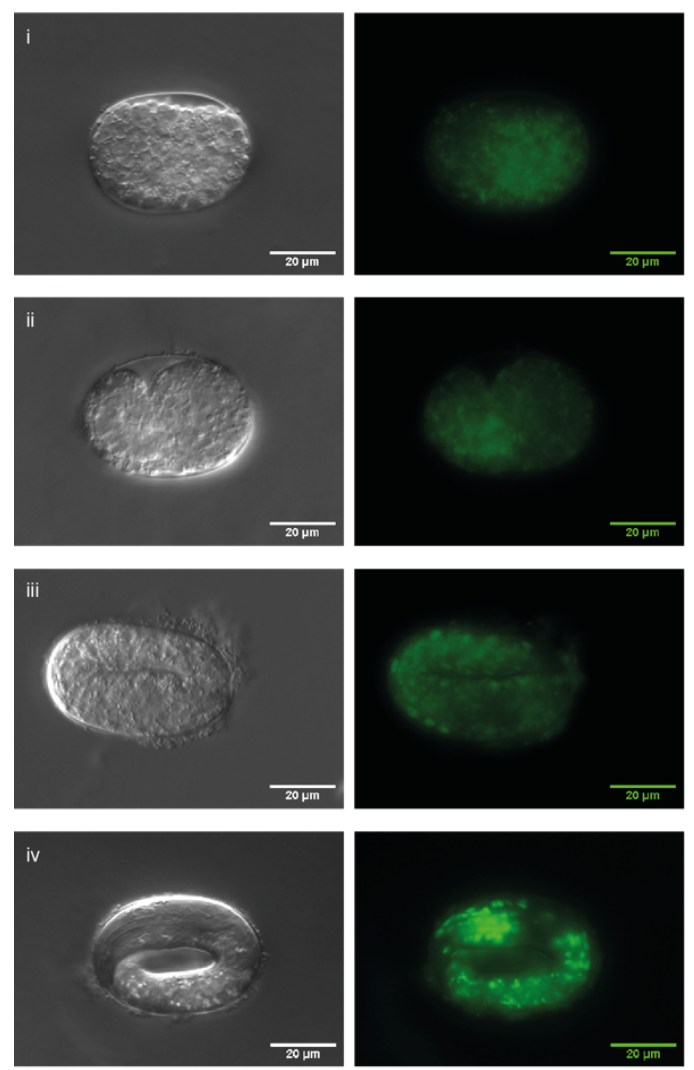

Figure S6: Related to Figure 5 - GFP-GRH-1 becomes detectable in elongating embryos

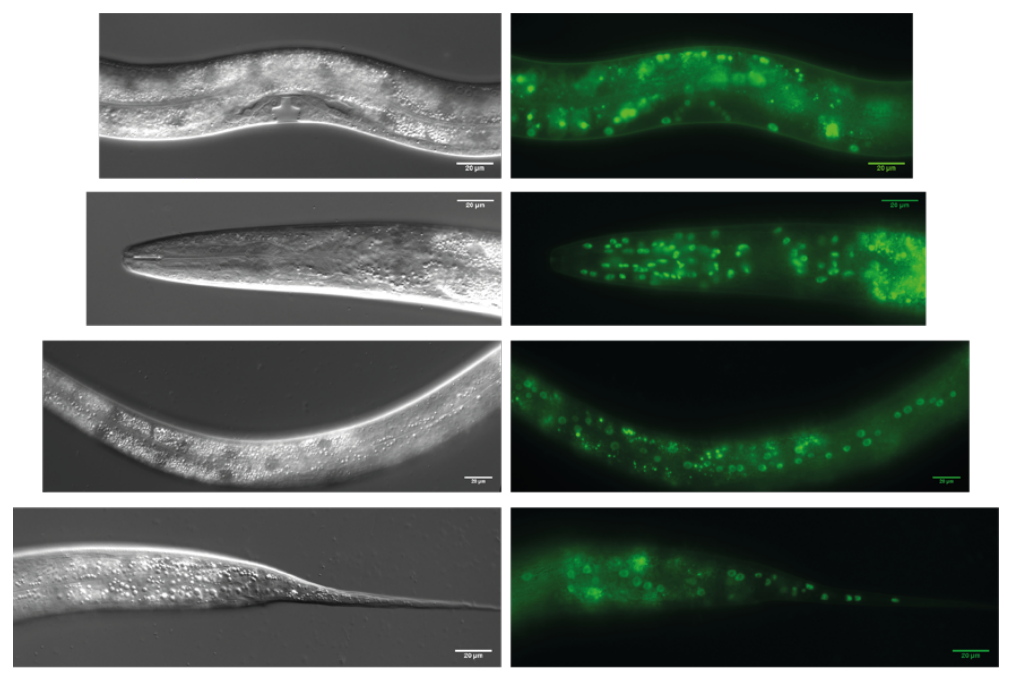

Figure S7: Related to Figure 5 - GFP-GRH-1 accumulates in various tissues during larval development 
bioRxiv preprint doi: https://doi.org/10.1101/2022.03.01.482504; this version posted March 4, 2022. The copyright holder for this preprint (which was not certified by peer review) is the author/funder, who has granted bioRxiv a license to display the preprint in perpetuity. It is made available under aCC-BY-NC 4.0 International license.

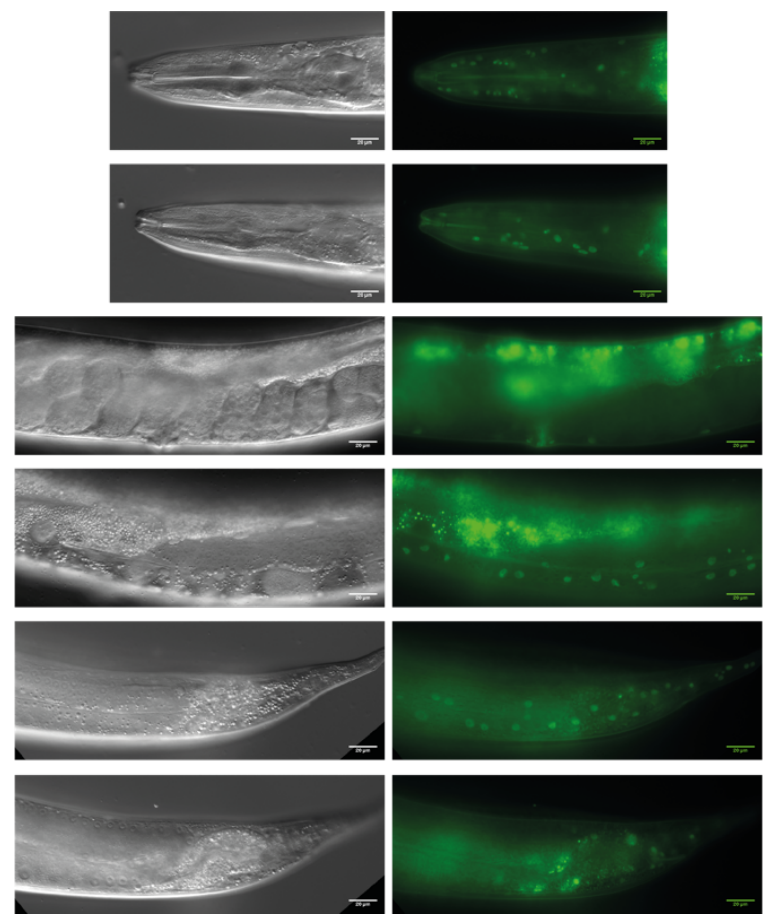

Figure S9: Related to Figure 5-GFP-GRH-1 accumulation is greatly reduced in adults

A

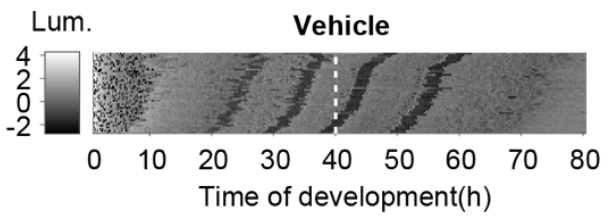

B

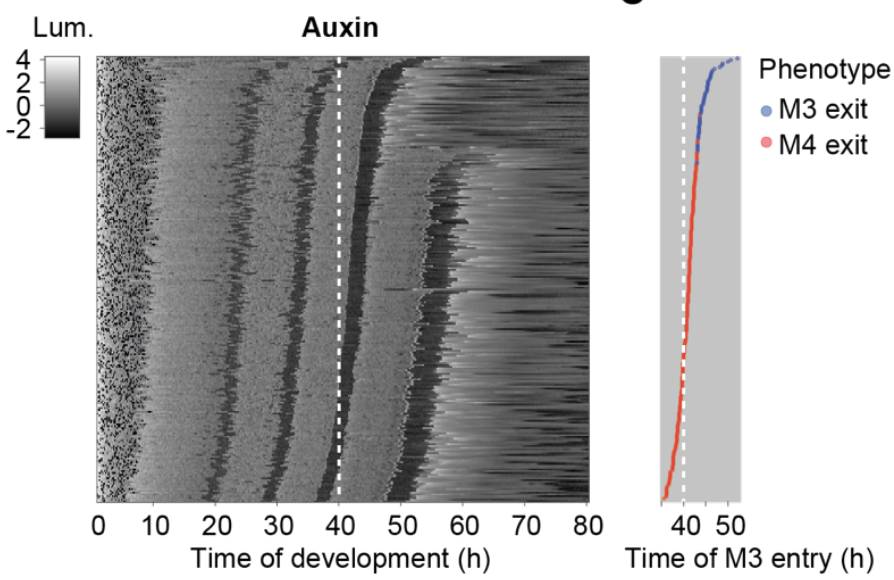

Figure S9: Related to Figure 6 - Molt exit phenotype is dependent on the time of GRH-1 depletion

A,B, Heatmap showing trend-correct luminescence (Lum) of grh-1(xe135); eft-3p::luc; eft-3p::TIR1 animals (HW2434) treated with vehicle $(0.25 \%$ ethanol, A) or $250 \mu \mathrm{M}$ auxin (B) at 40 hours (white dashed line). Black intensities correspond to low luminescence occurring during lethargus (molt). Embryos of various stages were left to hatch and develop during the assay. Luminescence traces are sorted by entry into molt 3 (M3) such that traces of early hatched animals are at the bottom and those of late hatched animals are at the top. 
bioRxiv preprint doi: https://doi.org/10.1101/2022.03.01.482504; this version posted March 4, 2022. The copyright holder for this preprint (which was not certified by peer review) is the author/funder, who has granted bioRxiv a license to display the preprint in perpetuity. It is made available under aCC-BY-NC 4.0 International license.

C, Quantification of time at which animals enter molt 2 ( $M 2$ entry). Animals were assigned to M3 exit phenotype (blue) or M4 exit phenotype (red) when M3 or M4 was the last observed molt in the luminescence trace respectively. Animals sorted according to B. 